TRANSACTIONS OF THE

AMERICAN MATHEMATICAL SOCIETY

Volume 352 , Number 10, Pages 4759-4787

S 0002-9947(00)02618-0

Article electronically published on June 14, 2000

\title{
AN EQUIVARIANT BRAUER SEMIGROUP AND THE SYMMETRIC IMPRIMITIVITY THEOREM
}

\author{
ASTRID AN HUEF, IAIN RAEBURN, AND DANA P. WILLIAMS
}

\begin{abstract}
Suppose that $(X, G)$ is a second countable locally compact transformation group. We let $\mathrm{S}_{G}(X)$ denote the set of Morita equivalence classes of separable dynamical systems $(A, G, \alpha)$ where $A$ is a $C_{0}(X)$-algebra and $\alpha$ is compatible with the given $G$-action on $X$. We prove that $\mathrm{S}_{G}(X)$ is a commutative semigroup with identity with respect to the binary operation $[A, G, \alpha][B, G, \beta]=\left[A \otimes_{X} B, G, \alpha \otimes_{X} \beta\right]$ for an appropriately defined balanced tensor product on $C_{0}(X)$-algebras. If $G$ and $H$ act freely and properly on the left and right of a space $X$, then we prove that $\mathrm{S}_{G}(X / H)$ and $\mathrm{S}_{H}(G \backslash X)$ are isomorphic as semigroups. If the isomorphism maps the class of $(A, G, \alpha)$ to the class of $(B, H, \beta)$, then $A \rtimes_{\alpha} G$ is Morita equivalent to $B \rtimes_{\beta} H$.
\end{abstract}

\section{INTRODUCTION}

Mackey's imprimitivity theorem identifies the unitary representations $U$ of a locally compact group $G$ which have been induced from a closed subgroup $H$. In Rieffel's formulation, it says that $U$ is induced precisely when it is part of a covariant representation $(\pi, U)$ of the dynamical system $\left(C_{0}(G / H), G\right)$; he proved the theorem by showing that the crossed product $C_{0}(G / H) \rtimes G$ is Morita equivalent to the group $C^{*}$-algebra $C^{*}(H)$ (and invented the $C^{*}$-algebraic theory of Morita equivalence while he was at it). The symmetric imprimitivity theorem of Green and Rieffel involves commuting free and proper actions of two groups, $G$ and $H$, on a space $X$, and asserts that $C_{0}(G \backslash X) \rtimes H$ is Morita equivalent to $C_{0}(X / H) \rtimes G$ (see [26]); one recovers Mackey's theorem by taking $H \subset G$ and $X=G$.

In recent years we have studied dynamical systems involving continuous-trace $C^{*}$-algebras $(A, G, \alpha)$ by viewing them as elements of an equivariant Brauer group $\operatorname{Br}_{G}(X)$ associated to the induced action of a group $G$ on the spectrum $X$ of the algebra $A[6]$, 18. Inspired by the symmetric imprimitivity theorem, it was shown in [16] that if $H$ and $G$ act freely and properly on $X$ as above, then there is a group isomorphism $\lambda$ of $\operatorname{Br}_{H}(G \backslash X)$ onto $\operatorname{Br}_{G}(X / H)$ such that $B \rtimes_{\beta} G$ is Morita equivalent to $A \rtimes_{\alpha} H$ whenever $(B, G, \beta)$ is a representative for $\lambda(A, H, \alpha)$. Green and Rieffel's symmetric imprimitivity theorem is recovered by taking $A=C_{0}(G \backslash X)$; in general, this Morita equivalence can be obtained by applying the noncommutative symmetric imprimitivity theorem of [21] to systems involving continuous-trace algebras.

Our present goal is to find a version of the isomorphism of [16] which incorporates as much algebraic structure as possible and yet gives the full strength of the

Received by the editors November 25, 1998.

2000 Mathematics Subject Classification. Primary 46L05, 46L35.

(C)2000 American Mathematical Society 
symmetric imprimitivity theorem as discussed in [21. We shall do this by introducing an equivariant Brauer semigroup $\mathrm{S}_{G}(X)$ : the objects in $\mathrm{S}_{G}(X)$ are again represented by systems $(A, G, \alpha)$, but now we insist only that $A$ is a $C_{0}(X)$-algebra in the sense of [14], 2], [17], and that the action $\alpha$ is compatible with a given action of $G$ on $X$ and $C_{0}(X)$. This incorporates many more systems: the algebras $A$ need not be type I or even nuclear, and their spectrums could be much bigger than $X$. (For example, any algebra of the form $C_{0}(X, D)$ could arise.)

We begin by introducing our Brauer semigroup $\mathrm{S}_{G}(X)$. As in [6], the product is based on a balanced tensor product of the underlying algebras. Here, however, we want to allow non-nuclear algebras, and this leads to technical difficulties: to get an associative multiplication we have to use the maximal tensor product rather than the spatial one. The objects in $\mathrm{S}_{G}(X)$ will be Morita equivalence classes of systems, and checking that these equivalences are compatible with our balanced tensor products requires some technical innovations.

Our basic isomorphism is proved in section 3. Specifically, we prove that if $G$ and $H$ are second countable locally compact groups acting freely and properly on the left and right, respectively, of a second countable locally compact space $X$ in such a way that $(s \cdot x) \cdot h=s \cdot(x \cdot h)$, then there is a semigroup isomorphism $\theta$ of $\mathrm{S}_{G}(X / H)$ onto $\mathrm{S}_{H}(G \backslash X)$. We give an explicit description of $\theta$ in terms of Rieffel's generalized fixed-point algebras $A^{\gamma}$ for proper actions $\gamma: G \rightarrow$ Aut $A$. Every class in $\mathrm{S}_{G}(X / H)$ has a representative of the form $\left(A^{\beta}, G, \bar{\alpha}\right)$, where $\alpha$ and $\beta$ are commuting proper actions of $G$ and $H$, respectively, on a $C_{0}(X)$-algebra $A$ which are compatible with the given actions on $X$. Since $A^{\beta}$ is a subalgebra of the multiplier algebra $M(A), \bar{\alpha}$ is just the natural lift of $\alpha$ to $M(A)$. The isomorphism $\theta$ maps the class of $\left(A^{\beta}, G, \bar{\alpha}\right)$ to the class of $\left(A^{\alpha}, H, \bar{\beta}\right)$. Furthermore, $A^{\alpha} \rtimes_{\bar{\beta}} H$ and $A^{\beta} \rtimes_{\bar{\alpha}} G$ are Morita equivalent. The Morita equivalence can be proved two ways; since $(A, G \times H, \alpha \times \beta)$ defines a class in $\mathrm{S}_{G \times H}(X)$ and the actions of $G$ and $H$ are free and proper, the equivalence follows from Kasparov's [14, Theorem 3.15] or from the second author's [21, Theorem 1.1]. Carefully untangling Kasparov's argument leads to a tensor product imprimitivity bimodule, and also to an interesting result concerning regular representations of the crossed products $A^{\alpha} \rtimes_{\bar{\beta}} H$ arising in our theorem. (Recall that regular representations are faithful exactly when the reduced crossed product $A^{\alpha} \rtimes_{\bar{\beta}, r} H$ equals the universal crossed product $A^{\alpha} \rtimes_{\bar{\beta}} H$.)

We present our result on regular representations in section 4 . Given that the action of $G$ on $X$ is free and proper, $\alpha: G \rightarrow$ Aut $A$ must be proper and saturated in Rieffel's sense and hence $A_{0}:=C_{c}(X) \cdot A$ can be completed to an $A \rtimes_{\alpha, r} G-A^{\alpha}$ imprimitivity bimodule $\mathrm{Y}^{\alpha}$ by [27, Theorem 1.5]. An important special case occurs when $A=C_{0}(X, D)$ and $\alpha=\tau \otimes \gamma$ for a $C^{*}$-dynamical system $\gamma: G \rightarrow \operatorname{Aut} D$. It is proved in [23, Theorem 2.1] that $C_{c}(X, D)$ can be completed to a $C_{0}(X, D) \rtimes_{\alpha}$ $G-\operatorname{Ind}_{G}^{X}(A, \alpha)$-imprimitivity bimodule $\mathrm{X}$. We prove that $\mathrm{Y}^{\alpha}$ is a quotient of $\mathrm{X}$, and use this to prove that regular representations of $A \rtimes_{\alpha} G$ are faithful; thus, $A \rtimes_{\alpha, r} G=A \rtimes_{\alpha} G$. Furthermore, $\mathrm{Y}^{\alpha}$ admits an $H$-action $u$ such that $\left(\mathrm{Y}^{\alpha}, u\right)$ implements an equivalence between $\left(A \rtimes_{\alpha} G, H, \beta \rtimes \iota\right)$ and $\left(A^{\alpha}, H, \bar{\beta}\right)$. Using [7], [5], we can form an $A \rtimes_{\alpha \times \beta}(G \times H)-A^{\alpha} \rtimes_{\bar{\beta}} H$-imprimitivity bimodule $Y^{\alpha} \rtimes_{u} H$ based on $C_{c}\left(H, \mathrm{Y}^{\alpha}\right)$. The set-up is symmetric in $H$ and $G$, and the tensor product bimodule

$$
\mathrm{W}:=\left(\mathrm{Y}^{\beta} \rtimes_{v} G\right)^{\sim} \otimes_{A \rtimes_{\alpha \times \beta}(G \times H)}\left(\mathrm{Y}^{\alpha} \rtimes_{u} H\right)
$$


is an $A^{\beta} \rtimes_{\bar{\alpha}} G-A^{\alpha} \rtimes_{\bar{\beta}} H$-imprimitivity bimodule. Using the symmetry in (1.1) and a result of Combes, we can prove that regular representations of $A^{\beta} \rtimes_{\bar{\alpha}} G$ are faithful if and only if regular representations of $A^{\alpha} \rtimes_{\bar{\beta}} H$ are. This generalizes a result of Quigg and Spielberg [20, Theorem 4.2].

Although the approach leading to (1.1) was used in proving our generalization of Quigg-Spielberg, it is often convenient in applications (cf. [9]) to have a more concrete realization of the imprimitivity bimodule. By appealing to [21], we can obtain an $A^{\beta} \rtimes_{\bar{\alpha}} G-A^{\alpha} \rtimes_{\bar{\beta}} H$-imprimitivity bimodule $\mathrm{Z}$ which is a quotient of the module constructed in [21, Theorem 1.1] based on $C_{c}(X, A)$. It is natural to ask if the two modules are the same, and it turns out that they are isomorphic. We give a proof in the case $A=C_{0}(X, D)$ at the end of section 4 .

Since $\operatorname{Br}_{G}(X)$ is easily identified with a subgroup of the group $\mathrm{S}_{G}(X)^{-1}$ of invertible elements in $\mathrm{S}_{G}(X)$, our definitions and results concerning $\mathrm{S}_{G}(X)$ extend those for the equivariant Brauer group $\operatorname{Br}_{G}(X)$. However, $\operatorname{Br}_{G}(X)$ is actually equal to $\mathrm{S}_{G}(X)^{-1}$, and we prove this in an appendix; the result and its proof are essentially due to Green [12, but were never published.

\section{Preliminaries}

2.1. $C_{0}(X)$-algebras. If $X$ is a locally compact (Hausdorff) space, then a $C_{0}(X)$ algebra is a $C^{*}$-algebra $A$ together with a homomorphism $\iota_{A}: C_{0}(X) \rightarrow Z M(A)$ which is nondegenerate in the sense that

$$
\iota_{A}\left(C_{0}(X)\right) \cdot A:=\operatorname{span}\left\{\iota_{A}(\varphi) \cdot a: \varphi \in C_{0}(X) \text { and } a \in A\right\}
$$

is dense in $A$. There is already considerable literature on $C_{0}(X)$-algebras; in particular, 3] and [17] give nice overall treatments. We recount some of the basic properties here for convenience.

The Dauns-Hofmann Theorem identifies the center $Z M(A)$ of the multiplier algebra with $C^{b}(\operatorname{Prim} A)$. It is not hard to see that if $\left(A, \iota_{A}\right)$ is a $C_{0}(X)$-algebra, then there is a continuous map $\sigma_{A}: \operatorname{Prim}(A) \rightarrow X$ such that $\iota_{A}(\varphi)$ and $\varphi \circ \sigma_{A}$ are equal in $Z M(A)$. Conversely, if $\sigma_{A}: \operatorname{Prim}(A) \rightarrow X$ is continuous, then

$$
\iota_{A}(\varphi) a:=\left(\varphi \circ \sigma_{A}\right) \cdot a \text { for all } \varphi \in C_{0}(X) \text { and } a \in A
$$

clearly defines a homomorphism $\iota_{A}: C_{0}(X) \rightarrow Z M(A)$, and it is not hard to see that $\iota_{A}$ is nondegenerate. When convenient, we can view $\sigma_{A}$ as a continuous function on $\hat{A}$. We usually suppress the homomorphism $\iota_{A}$, and write $\varphi \cdot a$ in place of $\iota_{A}(\varphi) a$ and $a \cdot \varphi$ in place of $a \iota_{A}(\varphi)$. Thus a $C_{0}(X)$-algebra $A$ is a central $C_{0}(X)$-bimodule 1 Conversely, if $A$ is a nondegenerate central $C_{0}(X)$-bimodule, then $\iota_{A}(\varphi) a:=\varphi \cdot a$ makes $A$ into a $C_{0}(X)$-algebra provided $(\varphi \cdot a)^{*}=\bar{\varphi} \cdot a^{*}$ for all $a \in A$ and $\varphi \in C_{0}(X)$.

We will write $C_{0, x}(X)$ for the ideal of functions in $C_{0}(X)$ which vanish at $x$. If $A$ is a $C_{0}(X)$-algebra, then let $I_{x}$ be the closed ideal $\overline{C_{0, x}(X) \cdot A}$ of $A$. Notice that if $x \notin \sigma_{A}(\operatorname{Prim}(A))$, then $I_{x}=A$; otherwise,

$$
I_{x}=\bigcap\left\{P: \sigma_{A}(P)=x\right\} .
$$

A $C_{0}(X)$-algebra can profitably be viewed as the upper-semicontinuous sections of a bundle over $X$ (cf., [10, [11], [15]). Specifically, the quotient $A / I_{x}$ will be called the fibre over $x$, and will be denoted by $A(x)$. The image of $a \in A$ in $A(x)$ will

\footnotetext{
${ }^{1}$ If $\mathrm{X}$ is a $B$-bimodule, we call $\mathrm{X}$ a central $B$-bimodule when $b \cdot x=x \cdot b$ for all $x \in \mathrm{X}$ and $b \in B$.
} 
be denoted by $a(x)$. Some of the basic properties of the "section" $x \mapsto a(x)$ are summarized in the next lemma.

Lemma 2.1 ([3], [17]). Suppose that $A$ is a $C_{0}(X)$-algebra.

(a) For all $a \in A$, the map $x \mapsto\|a(x)\|$ is upper semicontinuous. That is, $\{x \in$ $X:\|a(x)\| \geq \epsilon\}$ is closed for all $\epsilon \geq 0$.

(b) The map $x \mapsto\|a(x)\|$ is continuous for all $a \in A$ if and only if $\sigma_{A}$ is open from $\operatorname{Prim}(A)$ to $X$.

(c) For all $a \in A,\|a\|=\sup \{\|a(x)\|: x \in X\}$.

(d) If $\varphi \in C_{0}(X)$ and $a \in A$, then $(\varphi \cdot a)(x)=\varphi(x) a(x)$.

2.2. Balanced tensor products. We will write $\mathfrak{S}(X)$ for the collection of all separable $C_{0}(X)$-algebras. We want to define a $C_{0}(X)$-balanced tensor product on $\mathfrak{S}(X)$ with the objective of defining, up to isomorphism, an associative operation on $\mathfrak{S}(X)$ for which $C_{0}(X)$ is an identity. Since $\mathfrak{S}(X)$ can contain non-nuclear algebras, an approach based on identifying $\operatorname{Prim}(A \otimes B)$ with $\operatorname{Prim}(A) \times \operatorname{Prim}(B)$, such as employed in [23, §1], is inadequate. However, Blanchard's maximal $C_{0}(X)$-balanced tensor product is sufficient for our purposes [3], 2]. (Blanchard considered only the case in which $X$ is compact. However, his results extend easily to the general case. Additional details may be found in [11, §2].)

Definition 2.2 ([11, Definition 2.3]). Let $A$ and $B$ be two $C_{0}(X)$-algebras and let $I$ be the closed ideal of $A \otimes_{\max } B$ generated by

$$
\left\{a \cdot \varphi \otimes b-a \otimes \varphi \cdot b: a \in A, b \in B, \varphi \in C_{0}(X)\right\} .
$$

Then $A \otimes_{X} B:=\left(A \otimes_{\max } B\right) / I$, equipped with the $C_{0}(X)$-action given on the images $a \otimes_{X} b$ of elementary tensors $a \otimes b$ by

$$
\varphi \cdot\left(a \otimes_{X} b\right)=\varphi \cdot a \otimes_{X} b=a \otimes_{X} \varphi \cdot b,
$$

is called the maximal $C_{0}(X)$-balanced tensor product of $A$ and $B$.

Remark 2.3. It is possible to form other balanced tensor products, but our choice of the maximal tensor product is not a random one. For example, it is observed in [2] that Blanchard's minimal tensor product $\otimes_{C(X)}^{m}$, which is defined using the spatial tensor product, need not be associative. Such pathologies vanish when one of the factors is nuclear, and Definition 2.2 gives the same balanced tensor product used in 23] when at least one of the algebras is nuclear.

The universal property of the maximal tensor product implies that every representation of $A \otimes_{X} B$ is of the form $\pi_{A} \otimes \pi_{B}$, where $\pi_{A}$ and $\pi_{B}$ are commuting representations of $A$ and $B$, respectively, such that $\left.\bar{\pi}_{A}\right|_{C_{0}(X)}=\left.\bar{\pi}_{B}\right|_{C_{0}(X)}$ (cf., [11] Remark 2.5(a)]). If $A$ is both a $C_{0}(X)$ - and a $C_{0}(Y)$-algebra, and $C$ is a $C_{0}(Y)$ algebra, then $A \otimes_{Y} C$ is a $C_{0}(X)$-algebra in such a way that

$$
\varphi \cdot\left(a \otimes_{Y} c\right)=(\varphi \cdot a) \otimes_{Y} c \text { for } \varphi \in C_{0}(X) .
$$

If $\pi_{A}$ and $\pi_{C}$ is a pair of commuting representations such that

$$
\left.\bar{\pi}_{A}\right|_{C_{0}(Y)}=\left.\bar{\pi}_{C}\right|_{C_{0}(Y)}
$$

then the corresponding representation $\pi_{A} \otimes_{Y} \pi_{C}$ of $A \otimes_{Y} C$ satisfies

$$
\left.\left(\pi_{A} \otimes_{Y} \pi_{C}\right)^{-}\right|_{C_{0}(X)}=\left.\bar{\pi}_{A}\right|_{C_{0}(X)} .
$$


Lemma 2.4. Suppose that $X$ and $Y$ are second countable locally compact Hausdorff spaces, and that $p: X \rightarrow Y$ is a continuous surjection. Let $C \in \mathfrak{S}(Y)$, and $A, B \in \mathfrak{S}(X)$. Viewing, as necessary, elements in $\mathfrak{S}(X)$ as elements of $\mathfrak{S}(Y)$ via composition with $p$, there is an isomorphism $\Psi$ of $\left(A \otimes_{X} B\right) \otimes_{Y} C$ onto $\left(A \otimes_{Y} C\right) \otimes_{X} B$ such that

$$
\Psi\left(\left(a \otimes_{X} b\right) \otimes_{Y} c\right)=\left(a \otimes_{Y} c\right) \otimes_{X} b .
$$

Similarly, there are isomorphisms $\Psi_{1}: A \otimes_{X} B \rightarrow B \otimes_{X} A$ and $\Psi_{2}:\left(A \otimes_{X} B\right) \otimes_{Y}$ $C \rightarrow A \otimes_{X}\left(B \otimes_{Y} C\right)$ such that $\Psi_{1}\left(a \otimes_{X} b\right)=b \otimes_{X} a$ and $\Psi_{2}\left(\left(a \otimes_{X} b\right) \otimes_{Y} c\right)=$ $a \otimes_{X}\left(b \otimes_{Y} c\right)$.

Proof. The idea is to show that the natural isomorphism of $\left(A \otimes_{\max } B\right) \otimes_{\max } C$ onto $\left(A \otimes_{\max } C\right) \otimes_{\max } B$ (e.g., [25, Corollary B.29]) matches up the kernels of the quotient maps $q_{1}$ onto $\left(A \otimes_{X} B\right) \otimes_{Y} C$ and $q_{2}$ onto $\left(A \otimes_{Y} C\right) \otimes_{X} B$. The kernel of the quotient map $\left(A \otimes_{X} B\right) \otimes_{\max } C \rightarrow\left(A \otimes_{X} B\right) \otimes_{Y} C$ is

$$
\bigcap\left\{\operatorname{ker}\left(\pi_{A} \otimes_{X} \pi_{B}\right) \otimes \pi_{C}:\left(\pi_{A} \otimes_{X} \pi_{B}\right)^{-} \circ p^{*}=\left.\bar{\pi}_{C}\right|_{C_{0}(Y)}\right\} .
$$

So ker $q_{1}$, which is the inverse image of this ideal in $\left(A \otimes_{\max } B\right) \otimes_{\max } C$, is

$$
\bigcap\left\{\operatorname{ker}\left(\pi_{A} \otimes \pi_{B}\right) \otimes \pi_{C}:\left.\pi_{A}\right|_{C_{0}(X)}=\left.\pi_{B}\right|_{C_{0}(X)} \text { and }\left.\pi_{A}\right|_{C_{0}(X)} \circ p^{*}=\left.\pi_{C}\right|_{C_{0}(Y)}\right\} \text {. }
$$

On the other hand, the kernel of the map $\left(A \otimes_{Y} C\right) \otimes_{\max } B \rightarrow\left(A \otimes_{Y} C\right) \otimes_{X} B$ is

$$
\begin{aligned}
\bigcap\left\{\operatorname{ker}\left(\pi_{A} \otimes_{Y} \pi_{C}\right) \otimes \pi_{B}\right. & \left.:\left.\pi_{A} \otimes_{Y} \pi_{C}\right|_{C_{0}(X)}=\left.\pi_{B}\right|_{C_{0}(X)}\right\} \\
& =\bigcap\left\{\operatorname{ker}\left(\pi_{A} \otimes_{Y} \pi_{C}\right) \otimes \pi_{B}:\left.\pi_{A}\right|_{C_{0}(X)}=\left.\pi_{B}\right|_{C_{0}(X)}\right\} .
\end{aligned}
$$

Thus

$$
\begin{aligned}
& \operatorname{ker} q_{2}=\bigcap\left\{\operatorname{ker}\left(\pi_{A} \otimes \pi_{C}\right) \otimes \pi_{B}:\right. \\
& \left.\qquad\left.\pi_{A}\right|_{C_{0}(X)} \circ p^{*}=\left.\pi_{C}\right|_{C_{0}(Y)} \text { and }\left.\pi_{A}\right|_{C_{0}(X)}=\left.\pi_{B}\right|_{C_{0}(X)}\right\},
\end{aligned}
$$

and the assertions about $\Psi$ follow. The arguments for $\Psi_{1}$ and $\Psi_{2}$ are similar.

Corollary 2.5. The maximal $C_{0}(X)$-balanced tensor product is associative on $\mathfrak{S}(X)$.

2.3. Induced algebras. Suppose that $X$ is a free and proper left $G$-space and that $(A, G, \alpha)$ is a dynamical system. Then $\operatorname{Ind}_{G}^{X}(A, \alpha)$ is the $C^{*}$-subalgebra of $C^{b}(X, A)$ consisting of those functions $f$ such that

(a) $f(s \cdot x)=\alpha_{s}(f(x))$, and $\quad(b) x \mapsto\|f(x)\|$ defines an element of $C_{0}(G \backslash X)$.

When $X$ is a right $G$-space, condition $(a)$ is replaced by

$$
(a)^{\prime} f(x \cdot s)=\alpha_{s}^{-1}(f(x)) .
$$

An important example occurs when $G$ is a closed subgroup of a locally compact group $H$. If $H$ is viewed as a right $G$-space, then $\operatorname{Ind}_{G}^{H}(A, \alpha)$ was studied at length in [22]. Furthermore, if $\gamma=\tau \otimes \alpha$ is the diagonal action of $G$ on $C_{0}(X, A)$, given by

$$
\gamma_{s}(f)(x)=\alpha_{s}\left(f\left(s^{-1} \cdot x\right)\right)
$$

then $\operatorname{Ind}_{G}^{X}(A, \alpha)$ is the algebra denoted by $G C(X, A)^{\gamma}$ in [23 $\left.\S 2\right]$ and by $\operatorname{Ind}(A ; X, G, \alpha)$ or $\operatorname{Ind}(\alpha)$ in [21]. 
2.4. Proper actions on $C^{*}$-algebras. It is well-known that proper group actions on spaces - and therefore on abelian $C^{*}$-algebras — play a central rôle in the theory of crossed products. It is natural to try to extend the notion of proper actions to nonabelian $C^{*}$-algebras. Although it is not yet clear what the best definition of such an action should be, the notion developed by Rieffel in 27 is sufficient for our purposes 2 Let $(A, G, \alpha)$ be a dynamical system, and suppose that $A_{0}$ is a dense $\alpha$-invariant $*$-subalgebra of $A$. A multiplier $T \in M(A)$ belongs to $M\left(A_{0}\right)$ if $T$ maps $A_{0}$ into itself. Since each $\alpha_{s}$ extends to an automorphism $\bar{\alpha}_{s}$ of $M(A)$, we can consider the $*$-subalgebra $M\left(A_{0}\right)^{\alpha}$ of $\bar{\alpha}$-invariant elements in $M\left(A_{0}\right)$.

Definition 2.6 (27, Definition 1.2]). A dynamical system $(A, G, \alpha)$ is called proper if there is a dense $\alpha$-invariant *-subalgebra $A_{0}$ of $A$ such that

(a) for all $a, b \in A_{0}$, the functions $s \mapsto{ }_{E}\langle a, b\rangle(s):=\Delta_{G}(s)^{-\frac{1}{2}} a \alpha_{s}\left(b^{*}\right)$ and $s \mapsto a \alpha_{s}\left(b^{*}\right)$ are in $L^{1}(G, A)$.

(b) For each $a, b \in A_{0}$, there is a (uniquely determined) element $\langle a, b\rangle_{D}$ in $M\left(A_{0}\right)^{\alpha}$ such that for all $c \in A_{0}$

$$
\int c \alpha_{s}\left(a^{*} b\right) d s=c\langle a, b\rangle_{D} .
$$

Viewing ${ }_{E}\langle\cdot, \cdot\rangle$ as taking values in $A \rtimes_{\alpha, r} G$, Rieffel showed that

$$
E_{0}:=\operatorname{span}\left\{{ }_{E}\langle a, b\rangle: a, b \in A_{0}\right\}
$$

was an ideal in $A \rtimes_{\alpha, r} G$. The action $\alpha$ is called saturated if the closure $E$ of $E_{0}$ is all of $A \rtimes_{\alpha, r} G$. The closure in $M(A)$ of

$$
D_{0}:=\operatorname{span}\left\{\langle a, b\rangle_{D}: a, b \in A_{0}\right\}
$$

is defined to be the generalized fixed-point algebra of $\alpha$, and is denoted $A^{\alpha}$.

Of course, $A_{0}$ is an $E_{0}-D_{0}$-bimodule; the actions are given by

$$
{ }_{E}\langle a, b\rangle c=\int_{G} a \alpha_{s}\left(b^{*} c\right) d s \quad \text { and } \quad c\langle a, b\rangle_{D}=\int_{G} c \alpha_{s}\left(a^{*} b\right) d s
$$

for $a, b, c \in A_{0}$.

Theorem 2.7 ([27, Theorem 1.5]). Suppose that $(A, G, \alpha)$ is a proper dynamical system. Then the completion $\mathrm{Y}$ of $A_{0}$ is an $E-A^{\alpha}$-imprimitivity bimodule. In particular, if $\alpha$ is also saturated, then $A \rtimes_{\alpha, r} G$ is Morita equivalent to $A^{\alpha}$.

Suppose that $X$ is a left $G$-space. We will write $\tau^{G}: G \rightarrow \operatorname{Aut} C_{0}(X)$ for the associated automorphism group: $\tau_{s}^{G}(f)(x)=f\left(s^{-1} \cdot x\right)$. If $X$ is a right $H$-space, then $\tau_{h}^{H}(f)(x):=f(x \cdot h)$. (When there is no possibility of confusion, we will write $\tau$ in place of $\tau^{G}$ or $\tau^{H}$.)

Example 2.8 (27, Example 2.6]). Suppose $X$ is a free and proper left $G$-space, and $\alpha: G \rightarrow$ Aut $D$ is any $C^{*}$-dynamical system. Then the diagonal action $\tau \otimes \alpha$ is a proper and saturated action of $G$ on $A=C_{0}(X, D)$. Rieffel's generalized fixed point algebra $C_{0}(X, D)^{\tau \otimes \alpha}$ is $\operatorname{Ind}_{G}^{X}(D, \alpha)$.

\footnotetext{
${ }^{2}$ Rieffel has recently introduced a more general notion of properness for actions of locally compact groups on $C^{*}$-algebras [28]. However, the earlier version suffices for our purposes here, and the newer notion is not (yet) associated with a general Morita equivalence result.
} 
2.5. The Brauer Semigroup. Let $(G, X)$ be a second countable locally compact transformation group. We consider the class $\mathfrak{S}_{G}(X)$ of pairs $(A, \alpha)$ where $A$ is a $C_{0}(X)$-algebra and $\alpha: G \rightarrow$ Aut $A$ is a strongly continuous action which preserves the given $G$-action on $X$ in that

$$
\alpha_{s}(\varphi \cdot a)=\tau_{s}(\varphi) \cdot \alpha_{s}(a) \quad \text { for all } \varphi \in C_{0}(X), s \in G \text {, and } a \in A .
$$

Alternatively, we can replace (2.2) with $\sigma_{A}(s \cdot \pi)=s \cdot \sigma_{A}(\pi)$ for all $\pi \in \hat{A}$. Elements of $\mathfrak{S}_{G}(X)$ were called $G-C_{0}(X)$-algebras in [14.

Example 2.9 (Example 2.8 continued). The action $\varphi \cdot f=(\varphi \otimes 1) f$ makes $C_{0}(X, D)$ a $C_{0}(X)$-algebra, and $\left(C_{0}(X, D), \tau \otimes \alpha\right) \in \mathfrak{S}_{G}(X)$.

Recall that two systems $(A, G, \alpha)$ and $(B, G, \beta)$ are Morita equivalent if there is an $A-B$-imprimitivity bimodule $\mathrm{X}$ and a strongly continuous action $u$ of $G$ on $\mathrm{X}$ by linear maps such that for all $x, y \in \mathrm{X}$ and $s \in G$,

$$
{ }_{A}\left\langle u_{s}(x), u_{s}(y)\right\rangle=\alpha_{s}\left({ }_{A}\langle x, y\rangle\right), \quad \text { and } \quad\left\langle u_{s}(x), u_{s}(y)\right\rangle_{B}=\beta_{s}\left(\langle x, y\rangle_{B}\right) .
$$

Suppose that $(A, \alpha)$ and $(B, \beta)$ are elements in $\mathfrak{S}_{G}(X)$ which are Morita equivalent via $(\mathrm{X}, u)$. The actions of $A$ and $B$ extend to the respective multiplier algebras. Hence $\mathrm{X}$ becomes a $C_{0}(X)-C_{0}(X)$-bimodule. If $h_{\mathrm{X}}: \operatorname{Prim} B \rightarrow \operatorname{Prim} A$ is the Rieffel homeomorphism induced by $X$, and if we use the Dauns-Hofmann Theorem to identify $\iota_{A}(f)$ with $f \circ \sigma_{A}$ and $\iota_{B}(f)$ with $f \circ \sigma_{B}$, then it is not hard to see that

$$
\left(f \circ \sigma_{A}\right) \cdot x=x \cdot\left(f \circ \sigma_{B} \circ h_{\mathrm{X}}\right)
$$

(cf., 25, Proposition 5.7(a)]). It follows that we have $\iota_{A}(f) \cdot x=x \cdot \iota_{B}(f)$ for all $x \in \mathrm{X}$ and $f \in C_{0}(X)$ exactly when the diagram

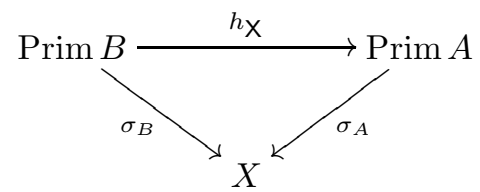

commutes. With these considerations in mind, we make the following definition.

Definition 2.10. Two elements $(A, \alpha)$ and $(B, \beta)$ in $\mathfrak{S}_{G}(X)$ are Morita equivalent over $X$ if they are Morita equivalent via $(\mathrm{X}, u)$ and if

$$
\iota_{A}(f) \cdot x=x \cdot \iota_{B}(f) \text { for all } f \in C_{0}(X) \text { and } x \in \mathrm{X} .
$$

In this event, we call $\mathrm{X}$ an $A-_{X} B$-imprimitivity bimodule.

Remark 2.11. We shall write $\mathfrak{B r}(X)$ for the elements $A$ in $\mathfrak{S}(X)$ with $\sigma_{A}$ a homeomorphism and $A$ continuous trace (so that we can identify $X, \hat{A}$, and $\operatorname{Prim} A$ ). If $A$ and $B$ belong to $\mathfrak{B r}(X)$ and if $\mathrm{X}$ is an $A{ }_{-} B$-imprimitivity bimodule, then it follows from (2.3) that $h_{\mathrm{X}}=$ id. In particular, the notions of Morita equivalence over $X$ and an $A{ }_{X} B$-imprimitivity bimodule defined above coincide on $\mathfrak{B r}(X)$ with those defined, for example, in [25, Definition 5.6], [24, §2] and [6, §1].

Lemma 2.12. Morita equivalence over $X$ is an equivalence relation on $\mathfrak{S}_{G}(X)$.

Proof. The proof follows the same lines as in [6] $\$ 3]$.

Definition 2.13. We let $\mathrm{S}_{G}(X)$ denote the set $\mathfrak{S}_{G}(X) / \sim$ of Morita equivalence classes over $X$. We call $\mathrm{S}_{G}(X)$ the equivariant Brauer semigroup. The class of $(A, \alpha)$ in $\mathrm{S}_{G}(X)$ is denoted by $[A, \alpha]$. 
Now suppose that $(A, \alpha)$ and $(B, \beta)$ belong to $\mathfrak{S}_{G}(X)$. Then $s \mapsto \alpha_{s} \otimes \beta_{s}$ is a strongly continuous automorphism group of $A \otimes_{\max } B$, and for all $a \in A, b \in B$, and $\varphi \in C_{0}(X)$

$$
\alpha_{s} \otimes \beta_{s}(\varphi \cdot a \otimes b-a \otimes \varphi \cdot b)=\tau_{s}(\varphi) \cdot a \otimes b-a \otimes \tau_{s}(\varphi) \cdot b
$$

It follows that each $\alpha_{s} \otimes \beta_{s}$ preserves the balancing ideal, and defines an automorphism $\alpha_{s} \otimes_{X} \beta_{s}$ of $A \otimes_{X} \beta$. It is not hard to check that $s \mapsto \alpha_{s} \otimes_{X} \beta_{s}$ is strongly continuous and equivariant; that is, $\left(A \otimes_{X} B, \alpha \otimes_{X} \beta\right) \in \mathfrak{S}_{G}(X)$.

Suppose that $\mathrm{X}$ is an $A-C$-imprimitivity bimodule and that $\mathrm{Y}$ is a $B-D$-imprimitivity bimodule. Then the algebraic tensor product $\mathrm{X} \odot \mathrm{Y}$ has a completion $\mathrm{X} \otimes_{\max } \mathrm{Y}$ which is an $A \otimes_{\max } B-C \otimes_{\max } D$-imprimitivity bimodule in the expected way. Although a sketch of this is given in [4, Proposition 2.9], we provide a proof for convenience.

Lemma 2.14. Suppose that $A, B, C$, and $D$ are $C^{*}$-algebras, that $\mathrm{X}$ is an $A-C$ imprimitivity bimodule, and that $\mathrm{Y}$ is a $B-D$-imprimitivity bimodule. Then $\mathrm{Z}:=$ $\mathrm{X} \odot \mathrm{Y}$ is an $A \odot B-C \odot D$-bimodule, and there are unique $A \odot B$ - and $C \odot D$-valued pre-inner products on $\mathbf{Z}$ such that

$$
\begin{aligned}
\langle\langle x \otimes y, z \otimes w\rangle\rangle & =\langle x, z\rangle \otimes_{A}\langle y, w\rangle \\
{ }_{A}\langle y \max B & \\
\langle\langle x \otimes y, z \otimes w\rangle\rangle_{C \otimes \max } D & =\langle x, z\rangle_{C} \otimes\langle y, w\rangle_{D} .
\end{aligned}
$$

With these inner products, $\mathrm{Z}$ becomes an $A \odot B-C \odot D$-pre-imprimitivity bimodule with respect to the maximal tensor product norms on $A \odot B$ and $C \odot D$. The completion $\mathrm{X} \otimes_{\max } \mathrm{Y}$ is an $A \otimes_{\max } B-C \otimes_{\max } D$-imprimitivity bimodule.

Proof. Exactly as in the proof of [25. Proposition 3.36], Z is easily seen to satisfy properties (a), (b), and (d) for a pre-imprimitivity bimodule as laid out in [25. Definition 3.9]. The issue is to show that the inner products are bounded; that is, we need to show that

$$
\begin{gathered}
\langle\langle\nu \cdot \alpha, \nu \cdot \alpha\rangle\rangle_{C \odot D} \leq\|\nu\|_{A \otimes_{\max } B}^{2}\langle\langle\alpha, \alpha\rangle\rangle_{C \odot D}, \quad \text { and } \\
\langle\langle\alpha \cdot \mu, \alpha \cdot \mu\rangle\rangle\|\| \mu \|_{C \otimes_{\max } D_{A \odot B}\langle\alpha \alpha, \alpha\rangle,}^{2}
\end{gathered}
$$

for all $\alpha \in \mathrm{X} \odot \mathrm{Y}, \mu \in C \odot D$, and $\nu \in A \odot B$. (We have decorated the inner products with the algebraic tensor products to emphasize that we are working on the incomplete bimodule $\mathrm{X} \odot \mathrm{Y}$.)

Let $\mathrm{X} \otimes_{\max } \mathrm{Y}$ be the completion of $\mathrm{X} \odot \mathrm{Y}$ as a right Hilbert $C \otimes_{\max } D$-module (see [25, Lemma 2.16]). Let $q: \mathrm{X} \odot \mathrm{Y} \rightarrow \mathrm{X} \otimes_{\max } \mathrm{Y}$ be the natural map. We claim that there is a homomorphism $\Phi_{A}$ of $M(A)$ into $\mathcal{L}\left(\mathrm{X} \otimes_{\max } \mathrm{Y}\right)$ such that $\Phi_{A}(m)(q(x \otimes y))=q(m \cdot x \otimes y)$. Since $\Phi_{A}\left(m^{*}\right)$ will act as an adjoint for $\Phi_{A}(m)$,

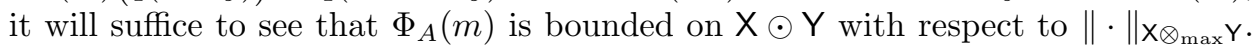
Since $M(A)$ is spanned by its unitary elements, we can assume that $m$ is unitary. 
If $\alpha=\sum_{i=1}^{n} x_{i} \otimes y_{i}$, then

$$
\begin{aligned}
\left\|\Phi_{A}(m) q(\alpha)\right\|_{\mathrm{X} \otimes_{\max } \mathrm{Y}}^{2} & :=\left\|\left\langle\Phi_{A}(m) q(\alpha), \Phi_{A}(m) q(\alpha)\right\rangle_{C \odot D}\right\| \\
& =\left\|\sum_{i, j=1}^{n}\left\langle m \cdot x_{i}, m \cdot x_{j}\right\rangle_{C} \otimes\left\langle y_{i}, y_{j}\right\rangle_{D}\right\| \\
& =\left\|\sum_{i, j=1}^{n}\left\langle x_{i}, x_{j}\right\rangle_{C} \otimes\left\langle y_{i}, y_{j}\right\rangle_{D}\right\| \\
& =\|q(\alpha)\|_{\mathrm{X} \otimes_{\max } \mathrm{Y}}^{2} ;
\end{aligned}
$$

this proves the claim.

Thus we obtain commuting homomorphisms $\Phi_{A}: A \rightarrow \mathcal{L}\left(\mathrm{X} \otimes_{\max } \mathrm{Y}\right)$ and $\Phi_{B}:$ $B \rightarrow \mathcal{L}\left(\mathrm{X} \otimes_{\max } \mathrm{Y}\right)$ such that $\Phi_{A}(a) q(x \otimes y)=q(a \cdot x \otimes y)$ and $\Phi_{B}(b) q(x \otimes y)=$ $q(x \otimes b \cdot y)$. The universal property of the maximal tensor product guarantees that there is a homomorphism $\Phi_{A} \otimes_{\max } \Phi_{B}$ of $A \otimes_{\max } B$ into $\mathcal{L}\left(\mathrm{X} \otimes_{\max } \mathrm{Y}\right)$. But

$$
\begin{aligned}
\langle\nu \cdot \alpha, \nu \cdot \alpha\rangle\rangle_{C \odot D} & =\langle q(\nu \cdot \alpha), q(\nu \cdot \alpha)\rangle_{C \otimes_{\max D}} \\
& =\left\langle\Phi_{A} \otimes_{\max } \Phi_{B}(\nu) q(\alpha), \Phi_{A} \otimes_{\max } \Phi_{B}(\nu) q(\alpha)\right\rangle_{C \otimes_{\max } D} \\
& \leq\left\|\Phi_{A} \otimes_{\max } \Phi_{B}(\nu)\right\|^{2}\langle q(\alpha), q(\alpha)\rangle_{C \otimes_{\max D}} \\
& \left.\leq\|\nu\|_{A \otimes_{\max } B}^{2}\langle\alpha, \alpha\rangle\right\rangle_{C \odot D} .
\end{aligned}
$$

This establishes (2.5), and (2.6) is proved similarly; there is a homomorphism $\Psi_{C} \otimes_{\max } \Psi_{D}$ of $C \otimes_{\max } D$ into the adjointable operators on the completion of $\mathrm{X} \odot \mathrm{Y}$ as a left Hilbert $A \otimes_{\max } B$-module. (Once we establish both (2.5) and (2.6), so that we know $Z$ is a pre-imprimitivity bimodule, then it follows that the two completions of $\mathrm{X} \odot \mathrm{Y}$ are the same.) $3.2]$.

The following lemma is a straightforward generalization of [6, Lemmas 2.1 and

Lemma 2.15. Suppose that $A, B, C, D \in \mathfrak{S}(X)$, that $\mathrm{X}$ is an $A-C$-imprimitivity bimodule over $X$, and that $Y$ is a $B$-D-imprimitivity bimodule over $X$. Then the Rieffel correspondence [25. Proposition 3.24] between ideals in $C \otimes_{\max } D$ and $A \otimes_{\max } B$ induced by $\mathrm{X} \otimes_{\max } \mathrm{Y}$ maps the balancing ideal $J_{X}$ of $C \otimes_{\max } D$ to the balancing ideal $I_{X}$ in $A \otimes_{\max } B$. In particular, the quotient $\mathrm{X} \otimes_{X} \mathrm{Y}$ of $\mathrm{X} \otimes_{\max } \mathrm{Y}$ is an $A \otimes_{X} B-C \otimes_{X} D$-imprimitivity bimodule over $X$.

Thus, if $(A, \alpha) \sim(C, \gamma)$ and $(B, \beta) \sim(D, \delta)$ in $\mathfrak{S}(X)$, then $\left(A \otimes_{X} B, \alpha \otimes_{X} \beta\right) \sim$ $\left(C \otimes_{X} D, \gamma \otimes_{X} \delta\right)$.

Proposition 2.16. The binary operation

$$
[A, \alpha][B, \beta]:=\left[A \otimes_{X} B, \alpha \otimes_{X} \beta\right]
$$

is well-defined on $\mathrm{S}_{G}(X)$, and with respect to this operation, $\mathrm{S}_{G}(X)$ is a commutative semigroup with identity equal to the class of $\left(C_{0}(X), \tau\right)$.

Proof. The operation is well-defined in view of Lemma 2.15 It is associative and commutative by Lemma 2.4 since the isomorphisms there are easily seen to be equivariant. The map $\varphi \otimes a \mapsto \varphi \cdot a$ extends to a well-defined $C_{0}(X)$-isomorphism of $C_{0}(X) \otimes_{X} A$ onto $A$, and the final assertion follows from this. 


\section{The Main Theorem}

Theorem 3.1. Suppose that $G$ and $H$ are second countable locally compact groups acting freely and properly on the left and right, respectively, of a second countable locally compact space $X$ in such a way that $(s \cdot x) \cdot h=s \cdot(x \cdot h)$. Then there is a semigroup isomorphism

$$
\theta: \mathrm{S}_{G}(X / H) \rightarrow \mathrm{S}_{H}(G \backslash X)
$$

such that if $[B, \beta]=\theta([A, \alpha])$, then $A \rtimes_{\alpha} G$ is Morita equivalent to $B \rtimes_{\beta} H$. Indeed, every class in $\mathrm{S}_{G}(X / H)$ has the form $\left[A^{\beta}, \bar{\alpha}\right]$ for some $(A, \alpha \times \beta) \in \mathfrak{S}_{G \times H}(X)$, and then we have

$$
\theta\left(\left[A^{\beta}, \bar{\alpha}\right]\right)=\left[A^{\alpha}, \bar{\beta}\right]
$$

so that $A^{\beta} \rtimes_{\bar{\alpha}} G$ is Morita equivalent to $A^{\alpha} \rtimes_{\bar{\beta}} H$.

Remark 3.2. In the next section, we will give another proof of the Morita equivalence of $A^{\beta} \rtimes_{\bar{\alpha}} G$ and $A^{\alpha} \rtimes_{\bar{\beta}} H$ which implies both are Morita equivalent to $A \rtimes_{\alpha \times \beta}(G \times H)$.

Lemma 3.3. Suppose that $A$ is a $C_{0}(X)$-algebra. Then the map $\varphi \otimes a \mapsto \varphi \cdot a$ extends to a homomorphism $\Phi$ of $C_{0}(X, A)$ onto $A$ which satisfies $\Phi(f)(x)=f(x)(x)$ for all $x \in X$ and $f \in C_{0}(X, A)$. The homomorphism $\Phi$ intertwines the action $\varphi \cdot f:=(\varphi \otimes 1) f$ of $C_{0}(X)$ on $C_{0}(X, A)$ with the given action on $A$.

Proof. The map $(\varphi, a) \mapsto \varphi \cdot a$ is bilinear, and hence induces a linear map $\Phi$ on the algebraic tensor product $C_{0}(X) \odot A$, which is easily seen to be a $*$-homomorphism. Let $\pi$ be a faithful representation of $A$. Because the range of $\iota: C_{0}(X) \rightarrow M(A)$ lies in $Z M(A)$, the representations $\pi$ and $\bar{\pi} \circ \iota$ have commuting ranges, and hence induce a representation $(\bar{\pi} \circ \iota) \otimes_{\max } \pi$ of $C_{0}(X) \otimes_{\max } A$. Since $\pi$ is faithful, it is isometric, and we have

$$
\begin{aligned}
\left\|\Phi\left(\sum_{i} \varphi_{i} \otimes a_{i}\right)\right\| & =\left\|\sum_{i} \varphi_{i} \cdot a_{i}\right\|=\left\|\pi\left(\sum_{i} \varphi_{i} \cdot a_{i}\right)\right\| \\
& =\left\|\sum_{i} \bar{\pi} \circ \iota\left(\varphi_{i}\right) \pi\left(a_{i}\right)\right\|=\left\|(\bar{\pi} \circ \iota) \otimes_{\max } \pi\left(\sum_{i} \varphi_{i} \otimes a_{i}\right)\right\| \\
& \leq\left\|\sum_{i} \varphi_{i} \otimes a_{i}\right\|_{\max } ;
\end{aligned}
$$

thus $\Phi$ is norm-decreasing and extends to a homomorphism on all of $C_{0}(X) \otimes_{\max } A$. We obtain the required map $\Phi$ by identifying $C_{0}(X) \otimes_{\max } A$ with $C_{0}(X, A)$ (cf., e.g., [25, Propositions B.43 and B.16]).

For an elementary tensor $f:=\varphi \otimes a$, the formula $\Phi(f)(x)=f(x)(x)$ follows from the identity $(\varphi \cdot a)(x)=\varphi(x) a(x)$ (Lemma 2.1 (d)), and this extends to general $f \in C_{0}(X, A)$ by linearity and continuity. The final remark is trivially true for $f \in C_{0}(X) \odot A$, and also extends to $f \in C_{0}(X, A)$ by linearity and continuity.

Proposition 3.4. Suppose $G$ acts freely and properly on $X$ and $(A, \alpha) \in \mathfrak{S}_{G}(X)$. Then $(A, \alpha)$ is proper with respect to the subalgebra

$$
A_{0}:=\operatorname{span}\left\{\varphi \cdot a: \varphi \in C_{c}(X) \text { and } a \in A\right\} .
$$


We have to verify the hypotheses (a) and (b) of Definition 2.6. For each pair of generators $\varphi \cdot a$ and $\psi \cdot b$, we have

$$
(\varphi \cdot a) \alpha_{s}(\psi \cdot b)^{*}=\left(\varphi \tau_{s}(\bar{\psi})\right) \cdot\left(a \alpha_{s}(b)^{*}\right) .
$$

The function $s \mapsto \varphi \tau_{s}(\bar{\psi})$ vanishes unless $\operatorname{supp} \varphi \cap s \cdot(\operatorname{supp} \psi) \neq \emptyset$, and hence has compact support because $G$ acts properly on $X$. Thus both functions $s \mapsto$ $(\varphi \cdot a) \alpha_{s}(\psi \cdot b)^{*}$ and ${ }_{E}\langle\varphi \cdot a, \psi \cdot b\rangle$ lie in $L^{1}(G, A)$, and (a) is satisfied.

To verify (b), we need a lemma.

Lemma 3.5. For each $f \in C_{c}(X, A)$, the function

$$
\mu(f)(x):=\int_{G} \tau_{s} \otimes \alpha_{s}(f)(x) d s=\int_{G} \alpha_{s}\left(f\left(s^{-1} \cdot x\right)\right) d s
$$

belongs to $\operatorname{Ind}_{G}^{X}(A, \alpha) \subset C^{b}(X, A) \subset M\left(C_{0}(X, A)\right)$. The multiplier $\bar{\Phi}(\mu(f))$ belongs to $M\left(A_{0}\right)^{\alpha}$, depends only on the image $\Phi(f)$ of $f$ in $A$, and satisfies

$$
c \bar{\Phi}(\mu(f))=\int_{G} c \alpha_{s}(\Phi(f)) d s \quad \text { for } c \in A_{0} .
$$

(In particular, the integrand on the right-hand side is a continuous function of compact support from $X$ to $A$, so the integral converges to an element of $A$.)

Proof. That $\mu(f) \in \operatorname{Ind}_{G}^{X}(A, \alpha)$ is proved for elementary tensors $f=\varphi \otimes a$ in 25 . Lemma 6.17], and the proof carries over almost verbatim. Since every multiplier $m$ of $A$ satisfies $(\varphi \cdot a) m=\varphi \cdot(a m)$ and $m \cdot(\varphi \cdot a)=\varphi \cdot(m a)$ for $\varphi \in C_{c}(X)$ and $a \in A$, we trivially have $\bar{\Phi}(\mu(f)) \in M\left(A_{0}\right)$. We shall next verify (3.2).

If $c=\varphi \cdot b \in A_{0}$, then

$$
(\varphi \cdot b) \bar{\Phi}(\mu(f))=\Phi(\varphi \otimes b) \bar{\Phi}(\mu(f))=\Phi((\varphi \otimes b) \mu(f)) .
$$

Now,

$$
((\varphi \otimes b) \mu(f))(x)=(\varphi(x) b) \int_{G} \alpha_{s}\left(f\left(s^{-1} \cdot x\right)\right) d s=\int_{G} \varphi(x) b \alpha_{s}\left(f\left(s^{-1} \cdot x\right)\right) d s .
$$

Because $G$ acts properly on $X, s \mapsto(\varphi \otimes b)\left(\tau_{s} \otimes \alpha_{s}(f)\right)$ is a continuous function of compact support with values in $C_{0}(X, A)$, and hence the integral $\int_{G}(\varphi \otimes b) \tau_{s} \otimes$ $\alpha_{s}(f) d s$ converges in $C_{0}(X, A)$ in such a way that

$$
\left(\int_{G}(\varphi \otimes b) \tau_{s} \otimes \alpha_{s}(f) d s\right)(x)=\int_{G} \varphi(x) b \alpha_{s}\left(f\left(s^{-1} \cdot x\right)\right) d s=(\varphi \otimes b) \mu(f)(x) .
$$

From the continuity of $\Phi$ we deduce that

$$
s \mapsto \Phi\left((\varphi \otimes b) \tau_{s} \otimes \alpha_{s}(f)\right)=(\varphi \cdot b) \alpha_{s}(\Phi(f))
$$

is a continuous function of compact support, and

$$
\begin{aligned}
(\varphi \cdot b) \bar{\Phi}(\mu(f)) & =\Phi((\varphi \otimes b) \mu(f)) \\
& =\int_{G} \Phi\left((\varphi \otimes b) \tau_{s} \otimes \alpha_{s}(f)\right) d s \\
& =\int_{G}(\varphi \cdot b) \alpha_{s}(\Phi(f)) d s
\end{aligned}
$$

as claimed. 
The formula (B.2) immediately implies that $\bar{\Phi}(\mu(f))$ depends only on $\Phi(f)$. To see that $\bar{\Phi}(\mu(f)) \in M\left(A_{0}\right)^{\alpha}$, we use that this integral formula converges in norm in $A$ :

$$
\begin{aligned}
c \alpha_{t}(\bar{\Phi}(\mu(f))) & =\alpha_{t}\left(\alpha_{t}^{-1}(c) \bar{\Phi}(\mu(f))\right) \\
& =\alpha_{t}\left(\int_{G} \alpha_{t}^{-1}(c) \alpha_{s}(\Phi(f)) d s\right) \\
& =\int_{G} c \alpha_{t s}(\Phi(f)) d s
\end{aligned}
$$

which is just $c \bar{\Phi}(\mu(f))$ by left-invariance of Haar measure.

Proof of Proposition 3.4. Given $\varphi \cdot a$ and $\psi \cdot b$ in $A_{0}$, we define $f \in C_{c}(X, A)$ by $f(x)=\overline{\varphi(x)} \psi(x) a^{*} b$ and we take for $\langle\varphi \cdot a, \psi \cdot b\rangle_{D}$ the multiplier $\bar{\Phi}(\mu(f))$ of $A_{0}$ described in Lemma 3.5. Since $\Phi(f)=(\varphi \cdot a)^{*}(\psi \cdot b)$, equation (3.2) implies that $\bar{\Phi}(\mu(f))$ has the property required in part (b) of Definition 2.6 Thus, $(A, \alpha)$ is proper.

With the definition of the inner product $\langle\cdot, \cdot\rangle_{D}$ fresh in our minds, we make some observations about the corresponding generalized fixed-point algebra $A^{\alpha}$. By definition, this is the $C^{*}$-subalgebra

$$
A^{\alpha}:=\overline{\operatorname{span}}\left\{\langle\varphi \cdot a, \psi \cdot b\rangle_{D}: \varphi, \psi \in C_{c}(X) \text { and } a, b \in A\right\}
$$

of $M(A)^{\alpha}$. The functions $\mu(f)$ for $f:=\bar{\varphi} \psi \otimes a^{*} b$ used to define $\langle\varphi \cdot a, \psi \cdot b\rangle_{D}$ span a $C_{0}(G \backslash X)$-submodule $M$ of $\operatorname{Ind}_{G}^{X}(A, \alpha)$, which has the property that, for each $a \in A$ and $x \in X$, there exists $g \in M$ satisfying $g(x)=a$ [25, Corollary 6.18]. A partition of unity argument on $G \backslash X$ shows that $M$ must therefore be dense in $\operatorname{Ind}_{G}^{X}(A, \alpha)$. Thus the continuity of $\bar{\Phi}$ implies that it maps $\operatorname{Ind}_{G}^{X}(A, \alpha)$ into $A^{\alpha}$. Further, since the image of $M$ is by definition dense in $A^{\alpha}$ and since $\bar{\Phi}$ has closed range, $\bar{\Phi}$ maps $\operatorname{Ind}_{G}^{X}(A, \alpha)$ onto $A^{\alpha}$. Thus:

Proposition 3.6. Suppose $G$ acts freely and properly on $X$ and $(A, \alpha) \in \mathfrak{S}_{G}(X)$. Then the map $\Phi$ of Lemma 3.3 induces a homomorphism $\bar{\Phi}$ of $\operatorname{Ind}_{G}^{X}(A, \alpha)$ onto $A^{\alpha}$.

Corollary 3.7. Suppose $G, H$, and $X$ are as in Theorem 3.1, and $(A, \alpha \times \beta) \in$ $\mathfrak{S}_{G \times H}(X)$. Then $\left(A^{\alpha}, \bar{\beta}\right) \in \mathfrak{S}_{H}(G \backslash X)$ and $\bar{\Phi}: \operatorname{Ind}_{G}^{X}(A, \alpha) \rightarrow A^{\alpha}$ is $C_{0}(G \backslash X)$ linear.

Proof. One can easily check that $\varphi \cdot\langle x, y\rangle_{D}=\langle\varphi \cdot x, y\rangle_{D}$ when $\varphi \in C_{0}(G \backslash X)$, so $A^{\alpha}$ is a $C_{0}(G \backslash X)$-submodule of $M(A)$. The action of $C_{0}(G \backslash X)$ on $\operatorname{Ind}_{G}^{X}(A, \alpha)$ is nondegenerate, and $\bar{\Phi}$ is easily seen to be $C_{0}(G \backslash X)$-linear, so the action on $A^{\alpha}=\bar{\Phi}\left(\operatorname{Ind}_{G}^{X}(A, \alpha)\right)$ is nondegenerate too. For $\varphi \otimes a \in C_{c}(X, A)$ we have

$$
\Phi\left(\tau_{h}^{H} \otimes \beta_{h}(\varphi \otimes a)\right)=\tau_{h}^{H}(\varphi) \cdot \beta_{h}(a)=\beta_{h}(\varphi \cdot a)=\beta_{h}(\Phi(\varphi \otimes a)),
$$

so $\bar{\Phi}$ intertwines the diagonal action of $\tau^{H} \otimes \beta$ on $\operatorname{Ind}_{G}^{X}(A, \alpha)$ with the action of $\bar{\beta}$ on $A^{\alpha}$. (We should write $\overline{\tau^{H} \otimes \beta}$, but this notation is awkward and we hope it will be clear from context that the action has been lifted to the multiplier algebra.) Since $H$ acts properly, $\tau^{H} \otimes \beta$ is strongly continuous on $\operatorname{Ind}_{G}^{X}(A, \alpha)(\operatorname{see} \operatorname{Lemma} 5.1)$. This implies both that $\bar{\beta}$ is strictly continuous on $A^{\alpha}$ and that $\bar{\beta}_{t}(\varphi \cdot m)=\tau_{t}^{H}(\varphi) \cdot \bar{\beta}_{t}(m)$ for $\varphi \in C_{0}(G \backslash X)$ and $m \in A^{\alpha}$. 
In [14, Kasparov uses a slightly different definition of a generalized fixed point algebra for a proper action; namely,

$$
\begin{aligned}
\left\{m \in M(A)^{\alpha}: \varphi \cdot m \in A \text { for all } \varphi \in C_{0}(X) \text { and }\left\|e_{i} m-m\right\| \rightarrow 0\right. \\
\text { as } \left.e_{i} \text { runs through an approximate identity for } C_{0}(G \backslash X)\right\} .
\end{aligned}
$$

Corollary 3.8 ([14 p. 164]). Suppose that $G$ acts freely and properly on $X$ and that $A \in \mathfrak{S}(X)$. Then Rieffel's generalized fixed point algebra $A^{\alpha}$ is given by (3.3).

Proof. If $m \in A^{\alpha}$, then $m=\bar{\Phi}(f)$ for some $f \in \operatorname{Ind}_{G}^{X}(A, \alpha)$ (Proposition [3.6). If $\varphi \in C_{0}(X)$, then $\varphi \cdot f \in C_{0}(X, A)$, and hence

$$
\varphi \cdot m=\varphi \cdot \bar{\Phi}(f)=\bar{\Phi}(\varphi \cdot f) \in \Phi\left(C_{0}(X, A)\right)=A .
$$

The assumption on approximate identities follows since $A^{\alpha}$ is a $C_{0}(G \backslash X)$-algebra.

Conversely, suppose that $m \in M(A)^{\alpha}$ satisfies $\varphi \cdot m \in A$ for all $\varphi \in C_{0}(X)$ and $\left\|e_{i} m \rightarrow m\right\| \rightarrow 0$. We may suppose that

$$
e_{i}(x)=\int_{G} \varphi_{i}\left(s^{-1} \cdot x\right) d s
$$

for some $\varphi_{i} \in C_{c}(X)$. Thus,

$$
m \sim e_{i} m=\left(\int_{G} \varphi_{i}\left(s^{-1} \cdot x\right) d s\right) \cdot m .
$$

As in the proof of Lemma 3.5 if we take $f=\varphi_{i} \otimes m$, then $\mu(f)=e_{i} \otimes m$ makes sense as a bounded continuous function of $X$ into $M(A)$ which is constant on $G$ orbits. This defines a multiplier of $C_{0}(X, A)$, so we can define $\bar{\Phi}(\mu(f))$. As in the proof of the lemma, if $c=\psi \cdot b \in C_{c}(X) \cdot A=A_{0}$, then because $\varphi_{i} \cdot m \in A$, the function $s \mapsto c \alpha_{s}\left(\varphi_{i} \cdot m\right)$ is continuous, has compact support, and satisfies

$$
c \bar{\Phi}(\mu(f))=\int c \alpha_{s}\left(\varphi_{i} \cdot m\right) d s
$$

Now we also have

$$
\begin{aligned}
(\psi \cdot b)\left(e_{i} \cdot m\right) & =\left(\psi e_{i}\right) \cdot(b m)=\Phi\left(\psi e_{i} \otimes b m\right)=\Phi(\psi \otimes b) \bar{\Phi}\left(e_{i} \otimes m\right) \\
& =\Phi(\psi \otimes b) \bar{\Phi}(\mu(f)),
\end{aligned}
$$

so

$$
(\psi \cdot b)\left(e_{i} \cdot m\right)=\int_{G}(\psi \cdot b) \alpha_{s}\left(\varphi_{i} \cdot m\right) d s
$$

We can factor $\varphi_{i}=\zeta \theta$ in $C_{c}(X)$, and then

$$
(\psi \cdot b)\left(\varphi_{i} \cdot m\right)=\int_{G}(\psi \cdot b) \alpha_{s}(\zeta \cdot(\theta \cdot m)) d s=\int_{G}(\psi \cdot b) \alpha_{s}(\Phi(\zeta \otimes \theta \cdot m)) d s .
$$

Now (3.2) implies that

$$
(\psi \cdot b)\left(e_{i} \cdot m\right)=(\psi \cdot b) \Phi(\mu(h)),
$$

where $h(x):=\zeta(x)(\theta \cdot m)$ is in $C_{c}(X, A)$. This is true for all $\psi \cdot b \in C_{c}(X) \cdot A=A_{0}$, so it implies $e_{i} \cdot m=\bar{\Phi}(\mu(b)) \in \bar{\Phi}\left(\operatorname{Ind}_{G}^{X}(A, \alpha)\right)=A^{\alpha}$. Since $A^{\alpha}$ is closed and $m \sim m \cdot e_{i}$, we deduce that $m \in A^{\alpha}$. 
Proposition 3.9. Let $G, H$ and $X$ be as in Theorem 3.1 Suppose that $(A, \alpha \times \beta) \in$ $\mathfrak{S}_{G \times H}(X)$ and that $p: X \rightarrow G \backslash X$ is the orbit map. Then $\tau^{G} \times \tau^{H} \otimes \bar{\beta}$ respects the balancing ideal and defines an action $\tau^{G} \times \tau^{H} \otimes_{G \backslash X} \bar{\beta}$ of $G \times H$ on $p^{*}\left(A^{\alpha}\right)$. The pull-back system $\left(p^{*}\left(A^{\alpha}\right), \tau^{G} \times \tau^{H} \otimes_{G \backslash X} \bar{\beta}\right)$ is covariantly isomorphic to $(A, \alpha \times \beta)$.

Proof. We start by showing that $A$ and $p^{*}\left(A^{\alpha}\right)$ are isomorphic. Let $J_{\Delta}$ be the balancing ideal for $p^{*}\left(A^{\alpha}\right)$. That is,

$$
p^{*}\left(A^{\alpha}\right)=C_{0}(X) \otimes_{C_{0}(G \backslash X)} A^{\alpha}:=C_{0}(X) \otimes A^{\alpha} / J_{\Delta} .
$$

As in Lemma 3.3 the maps $\varphi \otimes m \mapsto \varphi \cdot m$ and $\varphi \otimes f \mapsto \varphi \cdot f$ extend to homomorphisms $\Gamma: p^{*}\left(A^{\alpha}\right) \rightarrow A$ and $M: C_{0}(X) \otimes \operatorname{Ind}_{G}^{X}(A, \alpha) \rightarrow C_{0}(X, A)$, respectively. (Note that $\varphi \cdot m \in A$ by Corollary [3.8.) It is not difficult to see that $M$ is surjective. The surjectivity of $\Gamma$ is a consequence of the commutativity of the following diagram:

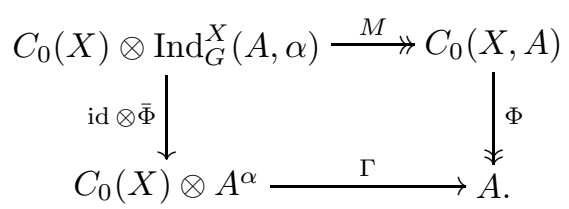

To see that $A$ and $p^{*}\left(A^{\alpha}\right)$ are isomorphic, it suffices to see that $\operatorname{ker}(\Gamma)=J_{\Delta}$. Let $q^{x}: A \rightarrow A(x)$ be the quotient map. Note that every irreducible representation $\pi$ of $A$ "lives" on the fibre $A\left(\sigma_{A}(\pi)\right)$; that is, $\pi=\hat{\pi} \circ q^{\sigma_{A}(\pi)}$ for some $\hat{\pi} \in A\left(\sigma_{A}(\pi)\right)^{\wedge}$. Since every irreducible representation of $\operatorname{Ind}_{G}^{X}(A, \alpha)$ is of the form $M^{(x, \pi)}$ where $M^{(x, \pi)}(F):=\pi(F(x))$ and $\pi \in \hat{A}$ ([25, Proposition 6.16]) and since $A^{\alpha}=\bar{\Phi}\left(\operatorname{Ind}_{G}^{X}(A, \alpha)\right)$, every irreducible representation $R$ of $A^{\alpha}$ must be (equivalent to one) such that $M^{(x, \pi)}=R \circ \bar{\Phi}$ for $(x, \pi) \in X \times \hat{A}$. Since $M^{(x, \pi)}(\operatorname{ker} \bar{\Phi})=\{0\}$, we must have $x=\sigma_{A}(\pi)$. It follows that every irreducible representation of $A^{\alpha}$ is of the form $\left.\bar{\pi}\right|_{A^{\alpha}}$ for some $\pi \in \hat{A}$. If $m \in A^{\alpha}$, then Corollary 3.8 implies that the multiplier $m(x)$ of $A(x)$ defined by $m$ actually belongs to $A(x)$. It follows that $\bar{\pi}(m)=\hat{\pi}\left(m\left(\sigma_{A}(\pi)\right)\right) 3$ Since $\left.\overline{s \cdot \pi}\right|_{A^{\alpha}}=\left.\bar{\pi}\right|_{A^{\alpha}}$, we have $\bar{\pi}(F(y))=\bar{\pi}\left(F\left(\sigma_{A}(\pi)\right)\right)$ for any $F \in C_{0}\left(X, A^{\alpha}\right)$ provided $p(y)=p\left(\sigma_{A}(\pi)\right)$. Thus [23, Lemma 1.1] implies that the norm of $F \in C_{0}\left(X, A^{\alpha}\right)$ in the quotient $p^{*}\left(A^{\alpha}\right)=C_{0}\left(X, A^{\alpha}\right) / J_{\Delta}$ is

$$
\left\|F+J_{\Delta}\right\|=\sup _{\pi \in \hat{A}}\left\|\bar{\pi}\left(F\left(\sigma_{A}(\pi)\right)\right)\right\|=\sup _{\pi \in \hat{A}}\left\|\hat{\pi}\left[F\left(\sigma_{A}(\pi)\right)\left(\sigma_{A}(\pi)\right)\right]\right\| .
$$

On the other hand, since $\Gamma(F)(x)=F(x)(x)$,

$$
\|\Gamma(F)\|=\sup _{\pi \in \hat{A}}\|\pi(\Gamma(F))\|=\sup _{\pi \in \hat{A}}\left\|\hat{\pi}\left[\Gamma(F)\left(\sigma_{A}(\pi)\right)\right]\right\|=\sup _{\pi \in \hat{A}}\left\|\hat{\pi}\left[F\left(\sigma_{A}(\pi)\right)\left(\sigma_{A}(\pi)\right)\right]\right\| .
$$

Therefore the kernel of $\Gamma$ is equal to $J_{\Delta}$, and $p^{*}\left(A^{\alpha}\right)$ and $A$ are isomorphic.

Since $\tau_{s}^{G} \times \tau_{h}^{H} \otimes \bar{\beta}_{h}$ certainly annihilates $\varphi \cdot \psi \otimes m-\varphi \otimes \psi \cdot m$ for all $\psi \in$ $C_{0}(G \backslash X), \varphi \in C_{0}(X)$, and $m \in A^{\alpha}$, it remains only to check that the isomorphism is equivariant. Since $(A, \alpha) \in \mathfrak{S}_{G}(X), \tau_{s}^{G}(\varphi) \bar{\alpha}_{s}(m)=\alpha_{s}(\varphi \cdot m)$ for all $\varphi \in C_{0}(X)$

\footnotetext{
${ }^{3}$ This analysis also allows us to identify $\left(A^{\alpha}\right)^{\wedge}$ with $G \backslash \hat{A}$. Note that $\left[\left.\bar{\pi}\right|_{A^{\alpha}}\right]=\left[\left.\bar{\eta}\right|_{A^{\alpha}}\right]$ in $\left(A^{\alpha}\right)^{\wedge}$ if and only if $M^{\left(\sigma_{A}(\pi), \pi\right)}$ and $M^{\left(\sigma_{A}(\eta), \eta\right)}$ are equivalent. Thus [25, Proposition 6.16] implies that the latter occurs exactly when $[\eta]=[s \cdot \pi]$ in $\hat{A}$.
} 
and $m \in M(A)$. Thus if $m \in A^{\alpha}$, then

$$
\begin{aligned}
\Gamma\left(\tau_{s}^{G} \times \tau_{h}^{H}(\varphi) \otimes \bar{\beta}_{h}(m)\right) & =\tau_{s}^{G} \times \tau_{h}^{H}(\varphi) \cdot \bar{\beta}_{h} \times \bar{\alpha}_{s}(m)=\beta_{h} \times \alpha_{s}(\varphi \cdot m) \\
& =\alpha_{s} \times \beta_{h}(\Gamma(\varphi \otimes m)) .
\end{aligned}
$$

The result follows.

Proposition 3.10. Let $G, H$, and $X$ be as in Theorem 3.1, and let $p: X \rightarrow G \backslash X$ be the orbit map. Suppose that $(B, \omega) \in \mathfrak{S}_{H}(G \backslash X)$. Then

$$
(A, \alpha \times \beta):=\left(p^{*}(B), \tau^{G} \times \tau^{H} \otimes_{G \backslash X} \omega\right)
$$

is in $\mathfrak{S}_{G \times H}(X)$ and $\left(A^{\alpha}, \bar{\beta}\right)$ is covariantly isomorphic to $(B, \omega)$.

Proof. We can identify $A(x)$ with $B(p(x))$. If $b \in B$, then the image of $1 \otimes b$ defines a multiplier $1 \otimes_{G \backslash X} b$ in $M(A)$ such that $\left(1 \otimes_{G \backslash X} b\right) a(x):=b(p(x)) a(x)$. The map $b \mapsto 1 \otimes_{G \backslash X} b$ is an isomorphism of $B$ into $M(A)$, and we will show that the image coincides with $A^{\alpha}$, where $\alpha$ is the action on $A=p^{*}(B)$ induced by $\tau^{G} \otimes 1$. Let $q: C_{0}(X, B) \rightarrow A$ be the quotient map. Then if $\psi \in C_{c}(X)$ and $b \in B$, $q(\psi \otimes b)(x)=\psi(x) b(p(x))$. Thus if $\varphi \in C_{c}(X)$, then $\varphi \otimes q(\psi \otimes b) \in C_{c}(X, A)$. Notice that

$$
\begin{aligned}
\alpha_{s}(q(\psi \otimes b))(x) & =q\left(\tau_{s}(\psi) \otimes b\right)(x)=\psi\left(s^{-1} \cdot x\right) b(p(x)) \\
& =q(\psi \otimes b)\left(s^{-1} \cdot x\right) .
\end{aligned}
$$

Lemma 6.17 of [25] implies that $\int_{G} \varphi\left(s^{-1} \cdot x\right) \tau_{s}^{G}(q(\psi \otimes b)) d s$ defines an element $F \in \operatorname{Ind}_{G}^{X}(A, \alpha)$; furthermore,

$$
\begin{aligned}
F(x)(y) & =\int_{G} \varphi\left(s^{-1} \cdot x\right) q(\psi \otimes b)\left(s^{-1} \cdot y\right) d s \\
& =\int_{G} \varphi\left(s^{-1} \cdot x\right) \psi\left(s^{-1} \cdot y\right) b(p(y)) d s \\
& =\int_{G} \varphi\left(s^{-1} \cdot x\right) \psi\left(s^{-1} \cdot y\right) d s \cdot b(p(y)) .
\end{aligned}
$$

Thus $\bar{\Phi}(F)=\zeta \cdot\left(1 \otimes_{G \backslash X} b\right)$, where $\zeta$ is the function in $C_{c}(G \backslash X)$ given by

$$
\zeta(p(x))=\int_{G} \varphi\left(s^{-1} \cdot x\right) \psi\left(s^{-1} \cdot x\right) d s .
$$

It follows from [25, Corollary 6.18] and a partition of unity argument on $G \backslash X$ that functions of the form

$$
\int_{G} \alpha_{s}\left(f\left(s^{-1} \cdot x\right)\right) d s \quad \text { for } f \in C_{c}(X, A)
$$

span a dense subspace of $\operatorname{Ind}_{G}^{X}(A, \alpha)$. It is clear that we may restrict $f$ to lie in a subspace of $C_{c}(X, A)$ which is dense in the inductive limit topology. In particular, functions of the form (3.4) span a dense subset of $\operatorname{Ind}_{G}^{X}(A, \alpha)$. Thus the range, $A^{\alpha}$, of $\bar{\Phi}$ restricted to $\operatorname{Ind}_{G}^{X}(A, \alpha)$ coincides with the image of $B$ as required.

It is clear that $\tau^{G} \times \tau^{H} \otimes \omega$ preserves the balancing ideal and defines an element $\left(p^{*}(B), \tau^{G} \times \tau^{H} \otimes_{G \backslash X} \omega\right)$ in $\mathfrak{S}_{G \times H}(X)$. Thus it will now suffice to see that $\bar{\beta}$ coincides with the action on the image of $B$ in $M(A)$ induced by $\omega$. However, $\beta$ is induced by $\tau^{H} \otimes \omega$, and since $\tau_{h}^{H} \otimes \omega_{h}(1 \otimes b)=1 \otimes \omega_{h}(b)$, the assertion is clear. 
In the proof of Theorem [3.1, it will be convenient to describe equivalence in $\mathfrak{S}_{G}(X)$ via linking algebras [25, Theorem 3.19]. The result we need is a version of the corollary in [5, §4] modified to accommodate the $C_{0}(X)$-action. If $(A, \alpha) \sim_{(\mathrm{X}, u)}$ $(B, \beta)$ in $\mathfrak{S}_{G}(X)$, then $A$ and $B$ are (isomorphic to) complementary full corners in the $C^{*}$-subalgebra of $\mathcal{L}(\mathrm{X} \oplus B)$ given by

$$
L:=\left\{\left(\begin{array}{cc}
a & x \\
b(y) & b
\end{array}\right): a \in A, b \in B, x \in \mathbf{X}, \text { and } y \in \mathbf{X}\right\}
$$

where $\widetilde{\mathrm{X}}$ is the $B-_{X} A$-imprimitivity bimodule dual to $\mathrm{X}$ and $b: \mathrm{X} \rightarrow \widetilde{\mathrm{X}}$ is the identity map (e.g., [25, pp. $49-50]$ ). Then $L$ is a $C_{0}(X)$-algebra:

$$
\varphi \cdot\left(\begin{array}{cc}
a & x \\
b(y) & b
\end{array}\right)=\left(\begin{array}{cc}
\varphi \cdot a & \varphi \cdot x \\
b(\bar{\varphi} \cdot y) & \varphi \cdot b
\end{array}\right),
$$

and there is a dynamical system $\gamma: G \rightarrow$ Aut $L$ such that

$$
\gamma_{s}\left(\begin{array}{cc}
a & x \\
b(y) & b
\end{array}\right)=\left(\begin{array}{cc}
\alpha_{s}(a) & u_{s}(x) \\
b\left(u_{s}(y)\right) & \beta_{s}(b)
\end{array}\right) .
$$

Since $u_{s}(a \cdot x)=\alpha_{s}(a) u_{s}(x)$ [25, Remark 7.3], it is not hard to check that $(L, \gamma) \in$ $\mathfrak{S}_{G}(X)$. The $C^{*}$-algebra $L$ is called the linking algebra, and $(L, \gamma)$ the linking system corresponding to $(A, \alpha) \sim_{(\mathrm{X}, u)}(B, \beta)$.

Lemma $3.11([5, \S 4])$. Suppose that $(A, \alpha)$ and $(B, \beta)$ belong to $\mathfrak{S}_{G}(X)$. Then $(A, \alpha) \sim(B, \beta)$ in $\mathfrak{S}_{G}(X)$ if and only if there is a $(L, \gamma) \in \mathfrak{S}_{G}(X)$ such that $A$ and $B$ are complementary full corners in $L$ and $\left.\gamma\right|_{A}=\alpha$ while $\left.\gamma\right|_{B}=\beta$.

Proof. The "only if" direction was outlined above. If $r$ and $s$ are full projections in $L$ such that $r+s=1_{L}, A=r L r$ and $B=s L s$, then $\mathrm{X}=r L s$ is an $A-_{X} B$ imprimitivity bimodule, and $u=\left.\gamma\right|_{r L s}$ implements an equivalence between $(A, \alpha)$ and $(B, \beta)$. Additional details can be found in [5, §4].

Proof of Theorem 3.1. We will produce an isomorphism $\theta: \mathrm{S}_{G \times H}(X) \rightarrow \mathrm{S}_{H}(G \backslash X)$ such that $\theta[A, \alpha \times \beta]=\left[A^{\alpha}, \bar{\beta}\right]$. Then, with the exception of the statements about Morita equivalence, the result will follow by symmetry. In order that $\theta$ be well-defined, we have to show $(A, \alpha \times \beta) \sim(B, \gamma \times \delta)$ in $\mathfrak{S}_{G \times H}(X)$ implies that $\left(A^{\alpha}, \bar{\beta}\right) \sim\left(B^{\gamma}, \bar{\delta}\right)$ in $\mathfrak{S}_{H}(G \backslash X)$. Let $(L, \Gamma \times \Delta)$ be the linking system for the given equivalence, and $r=\left(\begin{array}{cc}1_{A} & 0 \\ 0 & 0\end{array}\right) \in M(L)$ the projection associated to the corner $A$. If $\Phi^{L}: C_{0}(X, L) \rightarrow L$ is as in Lemma 3.3, and $\tilde{r} \in C^{b}(X, L)$ is the constant function $x \mapsto r$, then $\bar{\Phi}^{L}(\tilde{r} f)=r \bar{\Phi}^{L}(f)$ and $\bar{\Phi}^{L}(f \tilde{r})=\bar{\Phi}(f) r$ for all $f \in C^{b}(X, L)$. In fact, if $f \in \operatorname{Ind}_{G}^{X}(L, \Gamma)$, then both $\tilde{r} f$ and $f \tilde{r}$ also belong to $\operatorname{Ind}_{G}^{X}(L, \Gamma)$. Since $L^{\Gamma}=\bar{\Phi}_{G}^{L}\left(\operatorname{Ind}_{G}^{X}(L, \Gamma)\right)$, it follows that $r L^{\Gamma}$ and $L^{\Gamma} r$ are contained in $L^{\Gamma}$; thus $r \in M\left(L^{\Gamma}\right)$. Furthermore, $r L^{\Gamma} r=\bar{\Phi}_{G}^{L}\left(\tilde{r} \operatorname{Ind}_{G}^{X}(L, \Gamma) \tilde{r}\right)=$ $\bar{\Phi}_{G}\left(\operatorname{Ind}_{G}^{X}(A, \alpha)\right)=A^{\alpha}$. Since $\operatorname{Ind}_{G}^{X}(L, \Gamma) \tilde{r} \operatorname{Ind}_{G}^{X}(L, \Gamma)$ is dense in $\operatorname{Ind}_{G}^{X}(L, \Gamma)$, we also have $L^{\Gamma} r L^{\Gamma}=\bar{\Phi}_{G}^{L}\left(\operatorname{Ind}_{G}^{X}(L, \Gamma) \tilde{r} \operatorname{Ind}_{G}^{X}(L, \Gamma)\right)$ dense in $L^{\Gamma}$. That is, $A^{\alpha}=r L^{\Gamma} r$ is a full corner, and $B^{\gamma}=s L^{\Gamma} s$ is a complementary full corner, where $s=\left(\begin{array}{ll}0 & 0 \\ 0 & 1\end{array}\right)_{B}$. Since $\left.\bar{\Delta}\right|_{A^{\alpha}}=\bar{\beta}$ and $\left.\bar{\Delta}\right|_{B^{\gamma}}=\bar{\delta}$, we have $\left(A^{\alpha}, \bar{\beta}\right) \sim\left(B^{\gamma}, \bar{\delta}\right)$ in $\mathfrak{S}_{H}(G \backslash X)$ by Lemma 3.11, and $\theta$ is well-defined.

Next we want to define $\lambda: \mathrm{S}_{H}(G \backslash X) \rightarrow \mathrm{S}_{G \times H}(X)$ by $\lambda[B, \omega]:=\left[p^{*} B, \tau^{G} \times\right.$ $\left.\tau^{H} \otimes_{G \backslash X} \omega\right]$, and we need to see that this map is well-defined. Suppose that 
$(B, \omega) \sim(C, \zeta)$ in $\mathfrak{S}_{H}(G \backslash X)$, and let $\left(L_{1}, \Upsilon\right)$ be the corresponding linking system. Let

$$
(A, \alpha \times \beta):=\left(p^{*} L_{1}, \tau^{G} \times \tau^{H} \otimes_{G \backslash X} \Upsilon\right) \in \mathfrak{S}_{G \times H}(X)
$$

be as in Proposition [3.10. As above, it is not hard to verify that $p^{*} B$ and $p^{*} C$ are complementary full corners in $p^{*} L_{1}$ and that $\tau^{G} \times\left.\tau^{H} \otimes_{G \backslash X} \Upsilon\right|_{p^{*} B}=\tau^{G} \times \tau^{H} \otimes_{G \backslash X} \omega$, while $\tau^{G} \times\left.\tau^{H} \otimes_{G \backslash X} \Upsilon\right|_{p^{*} C}=\tau^{G} \times \tau^{H} \otimes_{G \backslash X} \zeta$. Thus $\lambda$ is well defined.

Proposition 3.9 and Proposition 3.10 imply that $\lambda=\theta^{-1}$. Since the inverse of a homomorphism is a homomorphism, it will suffice to see that $\lambda$ is a homomorphism. To do this, we proceed as on page 813 of 16 . We use Lemma 2.4 and the observation that the isomorphisms there are easily seen to be equivariant to justify the manipulations with tensor products:

$$
\begin{aligned}
& \lambda\left(B \otimes_{G \backslash X} C, \omega\right.\left.\otimes_{G \backslash X} \zeta\right)=\left(p^{*}\left(B \otimes_{G \backslash X} C\right), \tau^{G} \times \tau^{H} \otimes_{G \backslash X}\left(\omega \otimes_{G \backslash X} \zeta\right)\right) \\
&=\left(C_{0}(X) \otimes_{G \backslash X} B \otimes_{G \backslash X} C, \tau^{G} \times \tau^{H} \otimes_{G \backslash X} \omega \otimes_{G \backslash X} \zeta\right) \\
& \cong\left(C_{0}(X) \otimes_{X} C_{0}(X) \otimes_{G \backslash X} B \otimes_{G \backslash X} C,\right. \\
&\left.\tau^{G} \times \tau^{H} \otimes_{X} \tau^{G} \times \tau^{H} \otimes_{G \backslash X} \omega \otimes_{G \backslash X} \zeta\right) \\
& \cong\left(\left[C_{0}(X) \otimes_{G \backslash X} B\right] \otimes_{X}\left[C_{0}(X) \otimes_{G \backslash X} C\right],\right. \\
& {\left.\left[\tau^{G} \times \tau^{H} \otimes_{G \backslash X} \omega\right] \otimes_{X}\left[\tau^{G} \times \tau^{H} \otimes_{G \backslash X} \zeta\right]\right), }
\end{aligned}
$$

which is a representative for $\lambda[B, \omega] \lambda[C, \zeta]$.

Let $\bar{\Phi}_{G}$ be the restriction of $\bar{\Phi}$ to $\operatorname{Ind}_{G}^{X}(A, \alpha)$. It follows from Lemma 3.3 that

$$
\operatorname{ker} \bar{\Phi}_{G}=\left\{f \in \operatorname{Ind}_{G}^{X}(A, \alpha): f(x)(x)=0 \text { for all } x \in X\right\} \text {. }
$$

Recall that the $C_{0}(X)$-algebra structure on $A$ gives a continuous map $\sigma_{A}: \operatorname{Prim} A \rightarrow$ $X$ characterized by

$$
\sigma_{A}(P)=x \Longleftrightarrow I_{x} \subset P,
$$

so that $I_{x}=\bigcap\left\{P: \sigma_{A}(P)=x\right\}$. Thus

$$
\operatorname{ker} \bar{\Phi}_{G}=\left\{f \in \operatorname{Ind}_{G}^{X}(A, \alpha): f(x) \in P \text { whenever } \sigma_{A}(P)=x\right\}
$$

is the ideal $I\left(\sigma_{A}\right)$ considered in [21, $\left.\S 2\right]$. Because $\bar{\Phi}_{G}$ is surjective (Proposition 3.6) and intertwines the diagonal action $\tau^{H} \otimes \beta$ with $\bar{\beta}$, we deduce that

$$
\left(\operatorname{Ind}_{G}^{X}(A, \alpha) / I\left(\sigma_{A}\right)\right) \rtimes_{\tau^{H} \otimes \beta} H \cong A^{\alpha} \rtimes_{\bar{\beta}} H
$$

([13, Proposition 12]). In exactly the same way,

$$
\left(\operatorname{Ind}_{H}^{X}(A, \beta) / I\left(\sigma_{A}\right)\right) \rtimes_{\tau^{G} \otimes \alpha} G \cong A^{\beta} \rtimes_{\bar{\alpha}} G,
$$

and hence [21, Corollary 2.1] says that $A^{\alpha} \rtimes_{\bar{\beta}} H$ is Morita equivalent to $A^{\beta} \rtimes_{\bar{\alpha}} G$.

The Morita equivalence in the main theorem of [21] can be recovered from Theorem 3.1 .

Corollary 3.12 ([21] Theorem 1.1]). Suppose that X, $G$ and $H$ are as in Theorem 3.1. Also, suppose that $\alpha$ and $\beta$ are commuting actions of $G$ on a $C^{*}$-algebra D. As in Examples [2.8] and [2.9] $\left(C_{0}(X, D), \tau^{G} \otimes \alpha \times \tau^{H} \otimes \beta\right) \in \mathfrak{S}_{G \times H}(X)$. Thus

$$
\operatorname{Ind}_{G}^{X}(D, \alpha) \rtimes_{\tau^{H} \otimes \beta} H \quad \text { and } \quad \operatorname{Ind}_{H}^{X}(D, \beta) \rtimes_{\tau^{G} \otimes \alpha} G
$$

are Morita equivalent. 
Corollary 3.13. Let $X$ be a free and proper $G$-space. Suppose that $(A, \alpha) \in$ $\mathfrak{S}_{G}(X)$, and that $A$ has continuous trace. Then $A \rtimes_{\alpha} G$ has continuous trace.

Proof. Since $\operatorname{Ind}_{G}^{X}(A, \alpha)$ is a $C_{0}(G \backslash X)$-algebra, we can form the pull-back $p^{*}\left(\operatorname{Ind}_{G}^{X}(A, \alpha)\right)$ via the orbit map $p: C_{0}(X) \rightarrow C_{0}(G \backslash X)$. If $A$ has continuoustrace, then $C_{0}(X, A)$ certainly does and it is proved in [23 Proposition 3.7] that $p^{*}\left(\operatorname{Ind}_{G}^{X}(A, \alpha)\right)$ is isomorphic to $C_{0}(X, A)$. Therefore $\operatorname{Ind}_{G}^{X}(A, \alpha)$ has continuous trace by [22, Lemma 1.2]. Since $A^{\alpha}$ is the image of $\operatorname{Ind}_{G}^{X}(A, \alpha)$ under $\bar{\Phi}, A^{\alpha}$ has continuous trace. Since $A \rtimes_{\alpha} G$ is Morita equivalent to $A^{\alpha}$ by Theorem 3.1 (with $H=\{e\})$, the result follows from [29, Theorem 2.15].

Remark 3.14. Corollary 3.13 is a mild generalization of [22, Theorem 1.1(3)], where it is proved that $A \rtimes_{\alpha} G$ has continuous trace if $A$ has continuous trace and the action of $G$ on $\hat{A}$ is free and proper.

\section{Regular Representations}

In this section we want to investigate the question of when the universal norm and reduced norm coincide on crossed products of the type arising in Theorem 3.1 in other words, we want to determine circumstances in which regular representations of the crossed product are faithful. Recall that a regular representation of $A \rtimes_{\alpha} G$ is one induced from a faithful nondegenerate representation $\pi_{0}: A \rightarrow B(\mathcal{H})$. All such representations have the same kernel and provide faithful representations of the reduced crossed product $A \rtimes_{\alpha, r} G$ [19] Theorem 7.7.4]. The general question of the faithfulness of regular representations was considered by Quigg and Spielberg in 20], and systems for which the reduced norm equals the universal norm are sometimes called QS-regular. We shall see that their [20, Theorem 4.2] can be derived from our Theorem 3.1

The one-sided case - where $X$ is a free and proper left $G$-space, and $(A, \alpha) \in$ $\mathfrak{S}_{G}(X)$ - was treated by Kasparov in [14 Theorem 3.13]. We will use this result and a theorem of Combes to derive the main result of this section. Theorem 2.7 and Proposition 3.4 imply that $A_{0}=C_{c}(X) \cdot A$ can be completed to an $E{ }_{-G \backslash X} A^{\alpha_{-}}$ imprimitivity bimodule $\mathrm{Y}$, where $E$ is a subalgebra of $A \rtimes_{\alpha, r} G$. By definition, this subalgebra coincides with $A \rtimes_{\alpha, r} G$ when $\alpha$ is saturated.

Proposition 4.1. Suppose that $X$ is a free and proper $G$-space, and that $(A, \alpha) \in$ $\mathfrak{S}_{G}(X)$. Then regular representations of $A \rtimes_{\alpha} G$ are faithful, and

$$
A \rtimes_{\alpha} G=A \rtimes_{\alpha, r} G .
$$

Furthermore, $\alpha$ is saturated and $Y$ is an $A \rtimes_{\alpha} G-_{G \backslash X} A^{\alpha}$-imprimitivity bimodule.

The result on regular representations is to be expected since, once we have shown that $\alpha$ must be saturated, Rieffel's Theorem 2.7 implies that $A \rtimes_{\alpha, r} G$ is Morita equivalent to $A^{\alpha}$, while Theorem 3.1 implies that $A^{\alpha}$ is also Morita equivalent to $A \rtimes_{\alpha} G$. However a $C^{*}$-algebra and a proper quotient can be isomorphic (let alone Morita equivalent), so this observation only serves as motivation.

For our proof of the proposition, we shall realize Rieffel's module $Y$ as a quotient $X / M$, where $X$ is the imprimitivity bimodule described in [23, Theorem 2.2]; we now recall the formulas making $\mathrm{X}$ a $C_{0}(X, A) \rtimes_{\tau \otimes \alpha} G-\operatorname{Ind}_{G}^{X}(A, \alpha)$-imprimitivity bimodule. 
Let $\mathrm{X}_{0}:=C_{c}(X, A)$. If $z, w \in \mathrm{X}_{0}, f \in \operatorname{Ind}_{G}^{X}(A, \alpha)$, and $F \in C_{c}(G \times X, A)$, we can define inner products and actions on $\mathrm{X}_{0}$ by the following:

$$
\begin{aligned}
F \cdot z(x) & :=\int_{G} F(s, x) \alpha_{s}\left(z\left(s^{-1} \cdot x\right)\right) \Delta(s)^{\frac{1}{2}} d s, \\
z \cdot f(x) & :=z(x) f(x), \\
\langle z, w\rangle_{\operatorname{Ind}_{G}^{X}(A, \alpha)}(x) & :=\int_{G} \alpha_{s}\left(z\left(s^{-1} \cdot x\right)^{*} w\left(s^{-1} \cdot x\right)\right) d s, \quad \text { and } \\
C_{0}(X, A) \rtimes_{\tau \otimes \alpha} G & \langle z, w\rangle(s, x):=\Delta(s)^{-\frac{1}{2}} z(x) \alpha_{s}\left(w\left(s^{-1} \cdot x\right)\right)^{*} .
\end{aligned}
$$

Then [23, Theorem 2.2] implies that $\mathrm{X}_{0}$ can be completed to a $C_{0}(X, A) \rtimes_{\tau \otimes \alpha}$ $G-\operatorname{Ind}_{G}^{X}(A, \alpha)$-imprimitivity bimodule which we denote by $\mathrm{X}$.

Recall that the Rieffel correspondence establishes a bijection Ind : $\mathcal{I}(B) \rightarrow$ $\mathcal{I}(A)$ between the lattices of ideals of two Morita equivalent algebras $A$ and $B[25$. Proposition 3.24].

Lemma 4.2. Suppose that $G$ acts freely and properly on $X$. Let $\bar{\Phi}_{G}=\left.\bar{\Phi}\right|_{\operatorname{Ind}_{G}^{X}(A, \alpha)}$. If Ind $: \mathcal{I}\left(\operatorname{Ind}_{G}^{X}(A, \alpha)\right) \rightarrow \mathcal{I}\left(C_{0}(X, A) \rtimes_{\tau \otimes \alpha} G\right)$ is the Rieffel correspondence map for the imprimitivity bimodule $\mathrm{X}=\overline{\mathrm{X}_{0}}$, then $\operatorname{Ind}\left(\operatorname{ker} \bar{\Phi}_{G}\right)=(\operatorname{ker} \Phi) \rtimes_{\tau \otimes \alpha} G$.

Proof. To make the notation less cumbersome, let $J:=\operatorname{ker} \bar{\Phi}_{G}, K:=\operatorname{Ind}(J)$, and $C:=C_{0}(X, A) \rtimes_{\tau \otimes \alpha} G$. Recall that $K$ is the ideal generated by $\langle\mathrm{X} \cdot J, \mathrm{X}\rangle[25$ Proposition 3.24]. If $z \in C_{c}(X, A)$, then $z \cdot f \in C_{c}(X, A)$ for any $f \in J$. Since

$$
\operatorname{ker} \Phi=\left\{f \in C_{0}(X, A): f(x)(x)=0 \text { for all } x \in X\right\} \text {, }
$$

it is easy to see that

$$
{ }_{C}\left\langle C_{c}(X, A) \cdot J, C_{c}(X, A)\right\rangle \subseteq C_{c}(G, \operatorname{ker} \Phi) .
$$

Thus $K \subseteq \Phi \rtimes_{\gamma} G$.

For the other containment, let $\sigma_{A}: \hat{A} \rightarrow X$ be the continuous map giving the $C_{0}(X)$-algebra structure on $A$. By [25, Proposition 6.16], every irreducible representation of $\operatorname{Ind}_{G}^{X}(A, \alpha)$ is of the form $M^{(x, \pi)}$ where $M^{(x, \pi)}(F):=\pi(F(x))$. Then,

$$
J=\bigcap\left\{\operatorname{ker}\left(M^{(x, \pi)}\right): \pi \in \hat{A}, x \in X, \text { and } \sigma_{A}(\pi)=x\right\} .
$$

If $N^{(x, \pi)}$ is the representation of $C_{0}(X, A)$ given by $N^{(x, \pi)}(f):=\pi(f(x))$, then [23. p. 768$]$ implies that the representation of $C_{0}(X, A) \rtimes_{\tau \otimes \alpha} G$ induced from $M^{(x, \pi)}$ via $\mathrm{X}$ is equivalent to $\operatorname{Ind}_{\{e\}}^{G} N^{(x, \pi)}$, where the latter is the representation on $L^{2}\left(G, \mathcal{H}_{\pi}\right)$ given by

$$
\left[\operatorname{Ind}_{\{e\}}^{G} N^{(x, \pi)}(f)\right](\xi)(s)=\int_{G} \pi\left(\alpha_{s}^{-1}(f(t, s \cdot x))\right) \xi\left(t^{-1} s\right) d t
$$

Thus

$$
K=\bigcap\left\{\operatorname{ker}\left(\operatorname{Ind}_{\{e\}}^{G} N^{(x, \pi)}\right): \pi \in \hat{A}, x \in X, \text { and } \sigma_{A}(\pi)=x .\right\} .
$$

Since $(A, \alpha) \in \mathfrak{S}_{G}(X), \sigma_{A}\left(\pi \circ \alpha_{s}^{-1}\right)=s \cdot \sigma_{A}(\pi)$; thus it follows from (4.6) that $C_{c}\left(G, I_{\Delta}\right) \subseteq K$. This completes the proof. 
Remark 4.3. If $u: \mathrm{Z} \rightarrow \mathrm{W}$ is an isomorphism of right Hilbert $B$-modules, then $\Psi(T):=u T u^{-1}$ defines an isomorphism of $\mathcal{L}(\mathrm{Z})$ onto $\mathcal{L}(\mathrm{W})$. It is not hard to check that $\langle u(x), u(y)\rangle_{B}=\Psi\left(\langle x, y\rangle_{B}\right)$; hence $u$ induces an isomorphism of the imprimitivity algebras $\mathcal{K}(\mathrm{Z})$ and $\mathcal{K}(\mathrm{W})$.

Recall that if $\mathrm{X}$ is any Hilbert $B$-module and if $J$ is an ideal in $B$, then the quotient module $\mathrm{X}^{J}:=\mathrm{X} / \mathrm{X} \cdot J$ is a Hilbert $B / J$-module with respect to the quotient norm on $X^{J}$ [25, Proposition 3.25].

Proof of Proposition 4.1. Using (4.3) and Lemma 3.5. we see that

$$
\bar{\Phi}\left(\langle z, w\rangle_{\operatorname{Ind}_{G}^{X}(A, \alpha)}\right)=\langle\Phi(z), \Phi(w)\rangle_{D} \text { for all } z, w \in \mathbf{X}_{0} .
$$

It follows that $\alpha$ is saturated. Since the left and right $C_{0}(G \backslash X)$-actions on $\mathrm{Y}$ clearly coincide, $\mathrm{Y}$ is a $A \rtimes_{\alpha, r} G{ }_{-G \backslash X} A^{\alpha}$-imprimitivity bimodule. It remains only to show that $A \rtimes_{\alpha} G=A \rtimes_{\alpha, r} G$. Using (4.7), it follows that

$$
\|\Phi(x)\|_{\mathrm{Y}}:=\left\|\langle\Phi(x), \Phi(x)\rangle_{D}\right\|=\left\|\bar{\Phi}\left(\langle x, x\rangle_{\operatorname{Ind}_{G}^{X}(A, \alpha)}\right)\right\| \leq\left\|\langle x, x\rangle_{\operatorname{Ind}_{G}^{X}(A, \alpha)}\right\|=\|x\|_{\mathrm{X}} .
$$

Therefore the map $x \mapsto \Phi(x)$ is norm decreasing (as a map from $\mathrm{X}$ to $\mathrm{Y}$ ), and extends to a linear map $u: \mathrm{X} \rightarrow \mathrm{Y}$. Since

$$
\langle u(x), u(x \cdot b)\rangle_{D}=\langle u(x), u(y)\rangle_{D} \bar{\Phi}(b) \quad \text { for } x, y \in \mathrm{X}_{0} \text { and } b \in \operatorname{Ind}_{G}^{X}(A, \alpha),
$$

we have $X \cdot \operatorname{ker} \bar{\Phi}_{G} \subset \operatorname{ker} u$. (Recall that $\bar{\Phi}_{G}:=\left.\bar{\Phi}\right|_{\operatorname{Ind}_{G}^{X}(A, \alpha)}$.) Therefore we get an induced map $\bar{u}: \mathrm{X}^{\operatorname{ker} \bar{\Phi}_{G}} \rightarrow \mathrm{Y}$. If we identify $\operatorname{Ind}_{G}^{X}(A, \alpha) / \operatorname{ker} \bar{\Phi}_{G}$ with $A^{\alpha}$, then $\bar{u}$ is a Hilbert $A^{\alpha}$-module isomorphism. For convenience, we retain the notation from the proof of Lemma 4.2 $J=\operatorname{ker} \bar{\Phi}_{G}, K=\operatorname{Ind} J$, and $C=C_{0}(X, A) \rtimes_{\tau \otimes \alpha} G$. Note that $\mathrm{X}^{J}:=\mathrm{X} / \mathrm{X} \cdot J$ is a $C / K-\operatorname{Ind}_{G}^{X}(A, \alpha) / J$-imprimitivity bimodule [25. Proposition 3.25]. In view of Remark 4.3 and Lemma 4.2.

$$
{ }_{C / K}\langle x+\mathrm{X} \cdot J, y+\mathrm{X} \cdot J\rangle \mapsto{ }_{E}\langle u(x), u(y)\rangle
$$

defines an isomorphism of $C / K=C_{0}(X, A) \rtimes_{\tau \otimes \alpha} G / K$ onto $A \rtimes_{\alpha, r} G$. Since $C_{0}(X, A) \rtimes_{\tau \otimes \alpha} G / K=C_{0}(X, A) / \operatorname{ker} \Phi \rtimes_{\tau \otimes \alpha} G$ by Lemma 4.2 and 13, Proposition $12]$, and since $\Phi$ induces an equivariant isomorphism of $C_{0}(X, A) / \operatorname{ker} \Phi$ onto $A$, it follows that $C / K \cong A \rtimes_{\alpha} G$. Since this isomorphism is the identity on $C_{c}(G, A)$, the universal norm and the reduced norm coincide on $C_{c}(G, A)$.

Lemma 4.4. Let $X, G$, and $H$ be as in Theorem [3.1, and let $\mathrm{Y}$ be as in Proposition 4.1. Suppose that $(A, \alpha \times \beta) \in \mathfrak{S}_{G \times H}(X)$. Then there is a dynamical system $\beta \rtimes \iota: H \rightarrow \operatorname{Aut}\left(A \rtimes_{\alpha} G\right)$ such that $\beta \rtimes \iota_{h}(f)(s)=\beta_{h}(f(s))$ for $h \in H$ and $f \in C_{c}(G, A)$. Furthermore, $\left(A \rtimes_{\alpha} G, \beta \rtimes \iota\right)$ belongs to $\mathfrak{S}_{H}(G \backslash X)$, and is equivalent to $\left(A^{\alpha}, \bar{\beta}\right)$ via $(\mathrm{Y}, u)$, where $u_{h}(x)=\beta_{h}(x)$ for $h \in H$ and $x \in A_{0}$.

Proof. The assertions concerning $\beta \rtimes \iota$ are verified by routine computations as are the formulae

$$
\begin{gathered}
\left\langle u_{h}(x), u_{h}(y)\right\rangle(s)=\beta_{h}\left({ }_{E}\langle x, y\rangle\right)(s), \quad \text { and } \\
z\left\langle u_{h}(x), u_{h}(y)\right\rangle_{D}=\beta_{h}\left(\beta_{h}^{-1}(z)\langle x, y\rangle_{D}\right)=z \bar{\beta}_{h}\left(\langle x, y\rangle_{D}\right),
\end{gathered}
$$

where $x, y, z \in A_{0}$. Now it follows that $u$ extends to an action of $H$ on $\mathrm{Y}$ such that

$$
\left(A \rtimes_{\alpha} G, \beta \rtimes\right) \sim_{(\mathrm{Y}, u)}\left(A^{\alpha}, \bar{\beta}\right)
$$

as claimed. 
Suppose that $\pi_{0}: A \rightarrow B(\mathcal{H})$ is a nondegenerate representation. Let $\lambda^{G}$ denote the left-regular representation of $G$ on $L^{2}(G)$. Then the representation $\operatorname{Ind}_{e}^{G}\left(\pi_{0}, \iota\right)$ of $A \rtimes_{\alpha} G$ induced from $\pi_{0}$ is the integrated form of the covariant pair (Ind ${ }^{G} \pi_{0}, \lambda^{G} \otimes$ $\left.1_{\mathcal{H}}\right)$ on $L^{2}(G, \mathcal{H}) \cong L^{2}(G) \otimes \mathcal{H}$ given by

$$
\begin{aligned}
\left(\lambda^{G} \otimes 1_{\mathcal{H}}\right)_{s} \xi(r) & =\xi\left(s^{-1} r\right) \quad \text { and } \\
\left(\operatorname{Ind}^{G} \pi_{0}(a)\right) \xi(r) & =\pi_{0} \circ \alpha_{r}^{-1}(a) \xi(r) .
\end{aligned}
$$

When $\pi_{0}$ is faithful, $\operatorname{Ind}_{e}^{G}\left(\pi_{0}, \iota\right)$ is a faithful representation of the reduced crossed product $A \rtimes_{\alpha, r} G$ called a regular representation [19] Theorem 7.7.4].

Theorem 4.5. Suppose $X, G$, and $H$ are as in Theorem 3.1 and $(A, \alpha \times \beta) \in$ $\mathfrak{S}_{G \times H}(X)$. Then regular representations of $\left(A^{\alpha}, \bar{\beta}\right)$ are faithful if and only if regular representations of $\left(A^{\beta}, \bar{\alpha}\right)$ are faithful.

Proof. Assume that regular representations of $A^{\alpha} \rtimes_{\bar{\beta}} H$ are faithful. Let

$$
L: C_{c}(G \times H, A) \subset A \rtimes_{\alpha \times \beta}(G \times H) \rightarrow C_{c}\left(H, C_{c}(G, A)\right) \subset\left(A \rtimes_{\alpha} G\right) \rtimes_{\beta \rtimes_{\iota}} H
$$

be the natural $*$-homomorphism. If $(\pi, U \times V)$ is a covariant representation of $(A, G \times H)$, then a straightforward calculation shows that

$$
((\pi \rtimes U) \rtimes V) \circ L=\pi \rtimes(U \times V) .
$$

It follows immediately from (4.9) that $L$ is isometric for the universal norm and extends to an isomorphism of $A \rtimes_{\alpha \times \beta}(G \times H)$ onto $\left(A \rtimes_{\alpha} G\right) \rtimes_{\beta \rtimes \iota} H$.

Let $\pi_{0}: A \rightarrow B(\mathcal{H})$ be a faithful representation of $A$, and let $M$ be the regular representation $\operatorname{Ind}_{e}^{G \times H}\left(\pi_{0}, \iota\right)$ of $A \rtimes_{\alpha \times \beta}(G \times H)$. Note that $M$ is the integrated form of ( $\operatorname{Ind}^{G \times H} \pi_{0}, \lambda^{G \times H} \otimes 1_{\mathcal{H}}$ ). Let $\lambda^{G \times H} \otimes 1_{\mathcal{H}}=U \times V$, and write, abusing

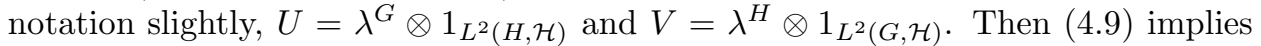
that $L$ intertwines $M$ with

$$
\left(\operatorname{Ind}^{G \times H} \pi_{0} \rtimes\left(\lambda^{G} \otimes 1_{L^{2}(H, \mathcal{H})}\right)\right) \rtimes\left(\lambda^{H} \otimes 1_{L^{2}(G, \mathcal{H})}\right) .
$$

Since $\operatorname{Ind}^{G \times H} \pi_{0} \rtimes\left(\lambda^{G} \otimes 1_{L^{2}(H, \mathcal{H})}\right)$ is equivalent to $\operatorname{Ind}^{H}\left(\operatorname{Ind}^{G} \pi_{0} \rtimes\left(\lambda^{G} \otimes 1_{\mathcal{H}}\right)\right)$, and since Proposition 4.1 implies that $\Pi:=\operatorname{Ind}^{G} \pi_{0} \rtimes\left(\lambda^{G} \otimes 1_{\mathcal{H}}\right)$ is a regular representation of $\left(A \rtimes_{\alpha} G\right) \rtimes_{\beta \rtimes \iota} H$, Lemma 4.4 and Combes's corollary in [5] $\left.\S 6\right]$ imply that $\Pi$ is faithful. Therefore, $M$ is faithful (and $A \rtimes_{\alpha \times \beta}(G \times H)$ is QSregular).

Now we can interchange the rôles of $G$ and $H$ and reverse the argument above to prove that regular representations of $A^{\beta} \rtimes_{\bar{\alpha}} G$ are faithful.

Corollary 4.6 ([20, Theorem 4.2]). Suppose that $(D, \alpha \times \beta) \in \mathfrak{S}_{G \times H}(X)$, and that $G, H$, and $X$ are as in Theorem 3.1. Then regular representations of $\operatorname{Ind}_{G}^{X}(D, \alpha)$ $\rtimes_{\tau^{H} \otimes \beta} H$ are faithful if and only if regular representations of $\operatorname{Ind}_{H}^{X}(D, \beta) \rtimes_{\tau^{G} \otimes \alpha} G$ are faithful.

Remark 4.7. Given commuting free and proper actions of $G$ and $H$ on $X$, it certainly may be the case that the action of $G \times H$ is neither free nor proper. But if $G \times H$ does act freely and properly, then regular representations of $A \rtimes_{\alpha \times \beta}(G \times H)$ are faithful, and the proof of Theorem 4.5 shows that regular representations of $A^{\alpha} \rtimes_{\bar{\beta}} H$ (and $A^{\beta} \rtimes_{\bar{\alpha}} G$ ) are always faithful. 
The proof of Theorem 4.5 suggests another proof of the Morita equivalence of $A^{\alpha} \rtimes_{\bar{\beta}} H$ and $A^{\beta} \rtimes_{\bar{\alpha}} G$ given in Theorem 3.1. First, recall that if two systems $(A, \alpha)$ and $(B, \beta)$ are Morita equivalent via $(\mathrm{X}, u)$, then the corresponding crossed products are Morita equivalent via an imprimitivity bimodule $\mathrm{X} \rtimes_{u} G$ which is the completion of $C_{c}(G, \mathrm{X})([\underline{5}, \S 6])$, equipped with $C_{c}(G, A)$ and $C_{c}(G, B)$ actions and inner products defined as follows. For $f \in C_{c}(G, A), g \in C_{c}(G, B)$, and $\xi, \eta \in C_{c}(G, \mathrm{X})$, let

$$
\begin{aligned}
f \cdot \xi(s) & =\int_{G} f(r) \cdot u_{r}\left(\xi\left(r^{-1} s\right)\right) d r \\
\xi \cdot g(s) & =\int_{G} \xi(r) \cdot \beta_{r}\left(g\left(r^{-1} s\right)\right) d r \\
\left\langle\rtimes_{\alpha} G\right. & \langle\xi, \eta\rangle(s)=\int_{G}^{A}\left\langle\xi(r), \Delta_{G}\left(s^{-1} r\right) u_{s}\left(\eta\left(s^{-1} r\right)\right)\right\rangle d r \\
\langle\xi, \eta\rangle_{B \rtimes_{\beta} G}(s) & =\int_{G} \beta_{r}^{-1}\left(\langle\xi(r), \eta(r s)\rangle_{B}\right) d r .
\end{aligned}
$$

Since Lemma 4.4 implies that $(\mathrm{Y}, u)$ implements an equivalence between $\left(A \rtimes_{\alpha} G, \beta \rtimes \iota\right)$ and $\left(A^{\alpha}, \bar{\beta}\right)$, it follows that $\left(A \rtimes_{\alpha} G\right) \rtimes_{\beta \rtimes_{\iota}} H$ (which we identify with $\left.A \rtimes_{\alpha \times \beta}(G \times H)\right)$ is Morita equivalent to $A^{\alpha} \rtimes_{\bar{\beta}} H$ via the $A \rtimes_{\alpha \times \beta}(G \times H)-A^{\alpha} \rtimes_{\bar{\beta}} H$ imprimitivity bimodule $\mathrm{Y} \rtimes_{u} H$. Similarly, $A \rtimes_{\alpha \times \beta}(G \times H)$ is Morita equivalent to $A^{\beta} \rtimes_{\bar{\alpha}} G$ via the imprimitivity bimodule $\mathrm{Y} \rtimes_{v} G$, where $v_{s}(x)=\alpha_{s}(x)$ for all $x \in A_{0}$. Therefore, we obtain an $A^{\beta} \rtimes_{\bar{\alpha}} G-A^{\alpha} \rtimes_{\bar{\beta}} H$-imprimitivity bimodule $\left(\mathrm{Y} \rtimes_{v} G\right)^{\sim} \otimes_{C}\left(\mathrm{Y} \rtimes_{u} H\right)$, where $C:=A \rtimes_{\alpha \times \beta}(G \times H)$ and $\left(\mathrm{Y} \rtimes_{v} G\right)^{\sim}$ is the dual bimodule.

The bimodule $\left(\mathrm{Y} \rtimes_{v} G\right)^{\sim} \otimes_{C}\left(\mathrm{Y} \rtimes_{u} H\right)$ is isomorphic to the module arising in the proof of Theorem 3.1 which is the quotient $Z^{\text {ker } \bar{\Phi}_{H} \rtimes_{\beta} H}$ of the $\operatorname{Ind}_{G}^{X}(A, \alpha) \rtimes_{\tau^{H} \otimes \beta}$ $H-\operatorname{Ind}_{H}^{X}(A, \beta) \rtimes_{\tau^{G} \otimes \alpha} G$-imprimitivity bimodule $\mathbf{Z}=\overline{C_{c}(X, A)}$ of the symmetric imprimitivity theorem [21, Theorem 1.1]. We give a proof of this only in the case $A=C_{0}(X, D)$ so that Rieffel's fixed point algebra is $\operatorname{Ind}_{G}^{X}(D, \alpha)$ (see Example 2.8). Here, Y equals the $C_{0}(X, D) \rtimes_{\tau^{G} \otimes \alpha} G-\operatorname{Ind}_{G}^{X}(D, \alpha)$-imprimitivity bimodule X described at the beginning of the section by equations (4.1)-4.4).

Lemma 4.8. Let $A=C_{0}(X, D)$. Also let $\mathrm{Z}$ be as above, and let $\mathrm{W}=\left(\mathrm{X} \rtimes_{v} G\right)^{\sim}$ $\otimes_{M}\left(\mathrm{X} \rtimes_{u} H\right)$, where $M=C_{0}(X, D) \rtimes_{\tau^{G} \otimes \alpha \times \tau^{H} \otimes \beta}(G \times H)$. The map $\Omega$ : b $\left(C_{c}(G \times X, D)\right) \odot C_{c}(H \times X, D) \rightarrow C_{c}(X, D)$ defined by

$$
\begin{aligned}
& \Omega(b(\xi) \otimes \eta)(x) \\
& =\int_{G} \int_{H} \alpha_{s^{-1}} \beta_{h^{-1}}\left(\xi\left(s, s \cdot x \cdot h^{-1}\right)^{*} \eta\left(h, s \cdot x \cdot h^{-1}\right)\right) \Delta_{H}(h)^{-\frac{1}{2}} \Delta_{G}(s)^{-\frac{1}{2}} d_{H}(h) d_{G}(s)
\end{aligned}
$$

extends to an imprimitivity-bimodule isomorphism $\Omega: \mathrm{W} \rightarrow \mathbf{Z}$.

Proof. Since $\Omega(b(\xi) \otimes \eta)$ clearly has compact support in $x$, to see that it belongs to $C_{c}(X, D)$ it will suffice to see that it is continuous on $X$. However, $\Omega(b(\xi) \otimes \eta)$ is of the form

$$
\int_{G} \int_{H} F(s, h, x) d_{H}(h) d_{G}(s)
$$

for some $F \in C_{c}(G \times H \times X, D)$, and the continuity follows from the uniform continuity of $F$. 
Now we check that $\Omega$ preserves the inner products. For ease of notation, we write $L:=\operatorname{Ind}_{H}^{X}(D, \beta) \rtimes_{\tau^{G} \otimes \alpha} G$ and $R:=\operatorname{Ind}_{G}^{X}(D, \alpha) \rtimes_{\tau^{H} \otimes \beta} H$. The bimodule Z is built as follows. If $L_{0}:=C_{c}\left(G, \operatorname{Ind}_{H}^{X}(D, \beta)\right)$ is viewed as a subalgebra of $L$ and if $R_{0}:=C_{c}\left(H, \operatorname{Ind}_{G}^{X}(D, \alpha)\right)$ is viewed as a subalgebra of $R$, then $\mathrm{Z}_{0}:=C_{c}(X, D)$ admits $L_{0}$ and $R_{0}$ actions and inner products defined as follows. For $b \in L_{0}$, $f, g \in \mathrm{Z}_{0}$, and $c \in R_{0}$,

$$
\begin{aligned}
b \cdot f(x) & =\int_{G} b(s, x) \Delta_{G}(s)^{\frac{1}{2}} \alpha_{s}\left(f\left(s^{-1} \cdot x\right)\right) d s \\
f \cdot c(x) & =\int_{H} \beta_{h}^{-1}\left(f\left(x h^{-1}\right) c\left(h^{-1}, x \cdot h^{-1}\right)\right) \Delta_{H}(h)^{-\frac{1}{2}} d h, \\
\langle f, g\rangle(s, x) & =\Delta_{G}(s)^{-\frac{1}{2}} \int_{H} \beta_{h}\left[f(x \cdot h) \alpha_{s}\left(g\left(s^{-1} \cdot x \cdot h\right)^{*}\right)\right] d h, \\
\langle f, g\rangle_{R}(h, x) & =\Delta_{H}(h)^{-\frac{1}{2}} \int_{G} \alpha_{t}\left(f\left(t^{-1} \cdot x\right)^{*} \beta_{h}\left(g\left(t^{-1} \cdot x \cdot h\right)\right)\right) d t .
\end{aligned}
$$

Using (4.16) and viewing $L_{0} \subset C_{c}\left(G, \operatorname{Ind}_{H}^{X}(D, \beta)\right) \subset C^{b}(G \times X, D)$,

$$
\begin{aligned}
&{ }_{L}\left\langle\Omega\left(b\left(\xi_{1}\right) \otimes \eta_{1}\right), \Omega\left(b\left(\xi_{2}\right) \otimes \eta_{2}\right)\right\rangle(s, x) \\
&=\Delta_{G}(s)^{-\frac{1}{2}} \int_{H} \beta_{h}\left[\Omega\left(b\left(\xi_{1}\right) \otimes \eta_{1}\right)(x \cdot h) \alpha_{s}\left(\Omega\left(b\left(\xi_{2}\right) \otimes \eta_{2}\right)\left(s^{-1} \cdot x \cdot h\right)\right)^{*}\right] d_{H}(h) \\
&=\int_{H} \int_{H} \int_{G} \int_{H} \int_{G} \beta_{h w^{-1}} \alpha_{r^{-1}}\left(\xi_{1}\left(r, r \cdot x \cdot h w^{-1}\right)^{*} \eta_{1}\left(w, r \cdot x \cdot h w^{-1}\right)\right) \\
& \beta_{h u^{-1}} \alpha_{s v^{-1}}\left(\eta_{2}\left(u, v s^{-1} \cdot x \cdot h u^{-1}\right)^{*} \xi_{2}\left(v, v s^{-1} \cdot x \cdot h u^{-1}\right)\right) \\
& v^{-1} r s \Delta_{H}(w u)^{-\frac{1}{2}} \Delta_{G}(s r v)^{-\frac{1}{2}} d_{G}(r) d_{H}(w) d_{G}(v) d_{H}(u) d_{H}(h)
\end{aligned}
$$

which, after interchanging the order of integration as necessary and performing the changes of variables, $v \mapsto v^{-1} r s, u \mapsto u^{-1} w$, and $h \mapsto h w$, equals

$$
\begin{aligned}
\int_{H} \int_{H} \int_{G} \int_{H} \int_{G} \beta_{h} \alpha_{r^{-1}}\left(\xi_{1}(r, r \cdot x \cdot h)^{*} \eta_{1}(w, r \cdot x \cdot h)\right) \\
\beta_{h u} \alpha_{r^{-1} v}\left(\eta_{2}\left(u^{-1} w, v^{-1} r \cdot x \cdot h u\right)^{*} \xi_{2}\left(v^{-1} r s, v^{-1} r \cdot x \cdot h u\right)\right) \\
\Delta_{G}(v)^{-\frac{1}{2}} \Delta_{H}\left(w u^{-1}\right) \Delta_{H}(u)^{\frac{1}{2}} d_{G}(r) d_{H}(w) d_{G}(v) d_{H}(u) d_{H}(h) .
\end{aligned}
$$

We aim to show that this coincides with the internal $L$-valued tensor product on $b\left(C_{c}(G \times X, D)\right) \odot C_{c}(H \times X, D) \subset \mathrm{W}$. Recall that $M:=C_{0}(X, D) \rtimes_{\tau^{G} \otimes \alpha \times \tau^{H} \otimes \beta}$ $(G \times H)$.

$$
\begin{aligned}
\left.{ }_{L}\left\langle b\left(\xi_{1}\right) \otimes \eta_{1}, b\left(\xi_{2}\right) \otimes \eta_{2}\right\rangle\right\rangle(s, x):={ }_{L}\left\langle b\left(\xi_{1}\right), b\left(\xi_{2}\right) \cdot{ }_{M}\left\langle\eta_{2}, \eta_{1}\right\rangle\right\rangle(s, x) \\
\quad={ }_{L}\left\langle b\left(\xi_{1}\right), b\left({ }_{M}\left\langle\eta_{1}, \eta_{2}\right\rangle \cdot \xi_{2}\right)\right\rangle(s, x) \\
=\left\langle\xi_{1},{ }_{M}\left\langle\eta_{1}, \eta_{2}\right\rangle \cdot \xi_{2}\right\rangle_{R}(s, x) \\
\quad \stackrel{(4.13)}{=} \int_{G} \tau_{r^{-1}}^{G} \otimes \alpha_{r^{-1}}\left(\left\langle\xi_{1}(r),{ }_{M}\left\langle\eta_{1}, \eta_{2}\right\rangle \cdot \xi_{2}(r s)\right\rangle_{\operatorname{Ind}_{H}^{X}(D, \beta)}\right)(x) d_{G}(r) \\
=\int_{G} \alpha_{r^{-1}}\left(\left\langle\xi_{1}(r),{ }_{M}\left\langle\eta_{1}, \eta_{2}\right\rangle \cdot \xi_{2}(r s)\right\rangle_{\operatorname{Ind}_{H}^{X}(D, \beta)}(r \cdot x)\right) d_{G}(r) \\
\stackrel{(4.3)}{=} \int_{G} \int_{H} \alpha_{r^{-1}} \circ \beta_{h}\left[\xi_{1}(r, r \cdot x \cdot h)^{*}{ }_{M}\left\langle\eta_{1}, \eta_{2}\right\rangle \cdot \xi_{2}(r s, r \cdot x \cdot h)\right] d_{H}(h) d_{G}(r)
\end{aligned}
$$


which, using (4.10) and then (4.1), and viewing $M \subset C_{c}\left(G, C_{c}\left(H, C_{0}(X, D)\right)\right) \subset$ $C_{c}(G \times H \times X, D)$, equals

$$
\begin{aligned}
& \int_{G} \int_{H} \int_{G} \int_{H} \alpha_{r^{-1}} \circ \beta_{h}\left[\xi_{1}(r, r \cdot x \cdot h)^{*}{ }_{M}\left\langle\eta_{1}, \eta_{2}\right\rangle(v, u, r \cdot x \cdot h)\right. \\
& \left.\quad \beta_{u} \circ \alpha_{v}\left(\xi_{2}\left(v^{-1} r s, v^{-1} r \cdot x \cdot h u\right)\right)\right] \Delta_{H}(u)^{\frac{1}{2}} d_{H}(u) d_{G}(v) d_{H}(h) d_{G}(r)
\end{aligned}
$$

which, now viewing $M \subset C_{c}\left(H, C_{c}\left(G, C_{0}(X, D)\right)\right)$ and using (4.12), is

$$
\begin{aligned}
& \int_{G} \int_{H} \int_{G} \int_{H} \int_{H} \alpha_{r^{-1}} \circ \beta_{h}\left[\xi_{1}(r, r \cdot x \cdot h)^{*}\right. \\
& \quad{ }^{C_{0}(X, D) \rtimes_{\tau}{ }^{G} \otimes \alpha}{ }^{G} \\
& \left.\quad \eta_{1}(w, \cdot), \Delta_{H}\left(u^{-1} w\right) \tau_{u}^{H} \otimes \beta_{u}\left(\eta_{2}\left(u^{-1} w, \cdot\right)\right)\right\rangle(v, r \cdot x \cdot h) \\
& \left.\quad \beta_{u} \circ \alpha_{v}\left(\xi_{2}\left(v^{-1} r s, v^{-1} r \cdot x \cdot h u\right)\right)\right] \Delta_{H}(u)^{\frac{1}{2}} d_{H}(u) d_{H}(w) d_{G}(v) d_{H}(h) d_{G}(r) .
\end{aligned}
$$

Using (4.4), the above is seen to coincide with the final formula for ${ }_{L}\langle\Omega(\cdot), \Omega(\cdot)\rangle$. A similar argument applies to the right-hand inner products.

To see that $\Omega$ is a bimodule map, we proceed using (4.14):

$$
\begin{aligned}
\Omega(f \cdot & (b(\xi) \otimes \eta))(x)=\Omega\left(b\left(\xi \cdot f^{*}\right) \otimes \eta\right)(x) \\
= & \int_{H} \int_{G} \alpha_{s^{-1}} \beta_{h^{-1}}\left(\xi \cdot f^{*}\left(s, s \cdot x \cdot h^{-1}\right)^{*} \eta\left(h, s \cdot x \cdot h^{-1}\right)\right) \\
= & \int_{H} \int_{G} \int_{G} \beta_{h^{-1}}\left(f\left(s^{-1} v, x \cdot h^{-1}\right)\right) \alpha_{s^{-1}} \beta_{h^{-1}}\left(\xi\left(v, s \cdot x \cdot h^{-1}\right)^{*} \eta\left(h, s \cdot x \cdot h^{-1}\right)\right) \\
& \Delta_{G}(s)^{-\frac{1}{2}} \Delta_{H}(h)^{-\frac{1}{2}} \Delta_{G}\left(s^{-1} v\right) d_{G}(v) d_{G}(s) d_{H}(h) \\
= & \int_{H} \int_{G} \beta_{G} \beta_{h^{-1}}\left(f\left(s, x \cdot h^{-1}\right)\right) \alpha_{s v^{-1}} \beta_{h^{-1}}\left(\xi\left(v, v s^{-1} \cdot x \cdot h^{-1}\right)^{*}\right. \\
= & \int_{H} \int_{G} \int_{G} f(s, x) \alpha_{s v^{-1}} \beta_{h^{-1}}\left(\xi\left(v, v s^{-1} \cdot x \cdot h^{-1}\right)^{*} \eta\left(h, v s^{-1} \cdot x \cdot h^{-1}\right)\right) \\
= & \int_{G} f(s, x) \alpha_{s}\left(\Omega(b(\xi) \otimes \eta)\left(s^{-1} \cdot x\right)\right) \Delta_{G}(s)^{\frac{1}{2}} d_{G}(s) \\
= & f \cdot \Omega(b(\xi) \otimes \eta)(x) .
\end{aligned}
$$

We omit the calculation showing that $\Omega$ respects the right action.

Now $\Omega$ extends to an isometric bimodule map $\Omega: \mathrm{W} \rightarrow \mathrm{Z}$ and $\Omega(\mathrm{W})$ is a closed $L-N$-subbimodule. Since $\overline{\langle\Omega(\mathrm{W}), \Omega(\mathrm{W})\rangle}=L$, we must have $\Omega(\mathrm{W})=\mathrm{Z}$ since the Rieffel correspondence is a bijection. This completes the proof.

\section{The Proof of the Symmetric Imprimitivity Theorem}

Our purpose here is to fix a minor gap in the proof of Lemma 1.2 of [21]. The gap occurs in equation (5): because the functions $g_{i}$ depend on the compact set $L$, when we pass to $m \geq m_{0}$, $\bigcup \operatorname{supp} g_{i}$ will increase and (5) may no longer hold. We thank Kevin Mansfield for drawing this to our attention.

To avoid this problem, we add to our index set $(N, L, \epsilon, j)$ a relatively compact open subset $U$ of $P$ such that $\pi(U) \supset L$, and add to the definition of $m=$ 
$(N, L, U, \epsilon, j) \geq m_{0}=\left(N_{0}, L_{0}, U_{0}, \epsilon_{0}, j_{0}\right)$ the requirement that $U \cap \pi^{-1}\left(L_{0}\right) \subset U_{0}$, where $\pi: P \rightarrow P / H$ is the orbit map. (So that, loosely speaking, the sets $U$ are getting thinner in the direction of the $H$-orbits, and stretching out along $P / H$.) Now we can proceed as in [26, p. 307], choosing the compact set $C$ to satisfy $C \subset U$ as well as $\pi(C) \supset L$, and the open covering $\left\{U_{i}\right\}$ to consist of open subsets $U_{i}$ of $U$. Then the functions $g_{i}$ will also satisfy $\operatorname{supp} g_{i} \subset U$.

In choosing $m_{0}$, we take $U_{0}$ to be a relatively compact open set such that $\pi\left(U_{0}\right) \supset$ $L_{0}$, and $j_{0}$ such that

$$
\left\|d_{j}^{2} b(k, p)-b(k, p)\right\|<\delta / 8 \quad \text { for } j \geq j_{0} \text { and }(k, p) \in\left(U_{0} \cap \pi^{-1}\left(L_{0}\right)\right) .
$$

Now if $m \geq m_{0}$, and both $g_{i}(p) \neq 0$ and $b(k, p) \neq 0$, then $p$ belongs to $U$ and to $\pi^{-1}\left(L_{0}\right)$, so that $(k, p) \in K \times\left(U \cap \pi^{-1}\left(L_{0}\right)\right) \subset K \times\left(U_{0} \cap \pi^{-1}\left(L_{0}\right)\right)$, and the estimate (5.1) applies.

We hope that the rest of the argument in 21] carries over.

Our proof of the strong continuity of the actions $\bar{\alpha}$ in Theorem 3.1 depends on the strong continuity of the diagonal action $\tau^{G} \otimes \alpha$ on $\operatorname{Ind}_{G}^{X}(A, \alpha)$. This continuity was used without comment in [21. We include a proof here for completeness.

Lemma 5.1. Suppose that we have commuting free and proper actions of locally compact groups $G$ and $H$ on a locally compact space $X$. Let $\alpha$ and $\beta$ be commuting actions of $G$ and $H$ on a separable $C^{*}$-algebra $A$. Then the diagonal actions $\tau^{G} \otimes \alpha$ : $G \rightarrow \operatorname{Aut}\left(\operatorname{Ind}_{H}^{X}(A, \beta)\right)$ and $\tau^{H} \otimes \beta: H \rightarrow \operatorname{Aut}\left(\operatorname{Ind}_{G}^{X}(A, \alpha)\right)$ are strongly continuous.

Proof. Let $\gamma=\tau^{G} \otimes \alpha$. Suppose that $t_{i} \rightarrow t$ in $G$. Since $\operatorname{Ind}_{H}^{X}(A, \beta)$ is a nondegenerate $C_{0}(X / H)$-module, functions $f$ for which $x H \mapsto\|f(x)\|$ has compact support $L$ are dense in $\operatorname{Ind}_{H}^{X}(A, \beta)$, and it is enough to prove that $\gamma_{t_{i}}(f) \rightarrow \gamma_{t}(f)$ for such $f$. Let $N$ be a compact neighborhood of $t$, and choose $i_{0}$ such that $i \geq i_{0}$ implies that $t_{i} \in N$. Then all the functions $x H \mapsto\left\|\gamma_{t_{i}}(f)(x)\right\|$ have support in $N L$. From the openness of the orbit map $p: X \rightarrow X / H$, we deduce that $N L=K H$ for a compact set $K \subset X$, and then for $i \geq i_{0}$, we have

$$
\left\|\gamma_{t_{i}}(f)-\gamma_{t}(f)\right\|=\sup _{x \in K}\left\|\gamma_{t_{i}}(f)(x)-\gamma_{t}(f)(x)\right\| .
$$

Now fix $\epsilon>0$, and choose $i_{1} \geq i_{0}$ such that $i \geq i_{1}$ implies that

$$
\left\|f\left(t_{i}^{-1} \cdot x\right)-f\left(t^{-1} \cdot x\right)\right\|<\epsilon / 2 \text { for all } x \in K,
$$

and $\left\|\alpha_{t_{i}}(a)-\alpha_{t}(a)\right\|<\epsilon / 2$ for all $a$ in the compact set $f\left(t^{-1} \cdot K\right)$. Then $i \geq i_{1}$ implies that $\left\|\gamma_{t_{i}}(f)-\gamma_{t}(f)\right\|<\epsilon$.

\section{Green's Theorem}

Theorem 6.1 (Green). The inclusion of $\mathfrak{B r}_{G}(X)$ into $\mathfrak{S}_{G}(X)$ defines an isomorphism of $\operatorname{Br}_{G}(X)$ onto the subgroup $\mathrm{S}_{G}(X)^{-1}$ of invertible elements in $\mathrm{S}_{G}(X)$.

We begin by showing that $\mathfrak{B r}_{G}(X)$ is saturated with respect to the equivalence relation in $\mathfrak{S}_{G}(X)$.

Lemma 6.2. Suppose that $(A, \alpha) \in \mathfrak{B r}_{G}(X)$ and that $(B, \beta) \in \mathfrak{S}_{G}(X)$. If $(B, \beta) \sim(A, \alpha)$, then $(B, \beta) \in \mathfrak{B r}_{G}(X)$, and $[A, \alpha]=[B, \beta]$ in $\operatorname{Br}_{G}(X)$. 
Proof. Let $\mathrm{X}$ be a $B-A$-imprimitivity bimodule over $X$. Then $B$ has continuous trace by [29, Theorem 2.15], and $h_{\mathrm{X}}$ is a homeomorphism of $\hat{A}=X$ onto $\hat{B}=$ Prim $B$ :

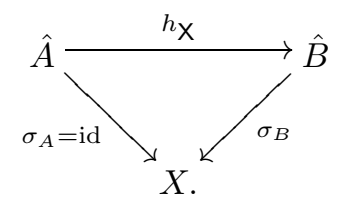

Since both $h_{\mathrm{X}}$ and $\sigma_{A}$ are homeomorphisms, so is $\sigma_{B}$. Thus, $(B, \beta) \in \mathfrak{B r}_{G}(X)$ as claimed.

In view of Remarks 2.11 and 2.3 the above inclusion gives a well-defined injective group-homomorphism $\Phi$ of $\operatorname{Br}_{G}(X)$ into $\mathrm{S}_{G}(X)^{-1}$. To complete the proof of Theorem 6.1, we only need to see that $\Phi$ is surjective. To do this, it will suffice to show that if $[A, \alpha]$ has an inverse in $\mathrm{S}_{G}(X)$, then $A$ has continuous trace and that $\sigma_{A}$ is a homeomorphism onto $X$. Since $[A, \alpha] \in \mathrm{S}_{G}(X)^{-1}$ certainly implies $[A] \in \mathrm{S}(X)^{-1}$, it suffices to show only that if $[A]$ is invertible in $\mathrm{S}(X)$, then $A$ is a continuous-trace $C^{*}$-algebra and that $\sigma_{A}$ is a homeomorphism.

By definition, $A$ has continuous trace if and only if the (not necessarily closed) ideal $m(A)$ of continuous trace elements is dense [8]. Recall that every $C^{*}$-algebra has a dense hereditary ideal $\kappa(A)$, which is minimal among all dense ideals 19 Theorem 5.6.1]. The ideal $\kappa(A)$ is called the Pedersen ideal of $A$. It can be constructed as follows. Let $C_{c}^{+}((0, \infty))$ be the set of compactly supported nonnegative functions on $(0, \infty)$. Put

$$
\kappa_{0}(A):=\left\{f(a): a \in A^{+} \text {and } f \in C_{c}^{+}((0, \infty))\right\} .
$$

Then $\kappa(A)$ is the ideal whose positive elements are exactly

$$
\kappa(A)^{+}:=\left\{a \in A^{+}: a \leq \sum_{i=1}^{n} a_{i} \text { with each } a_{i} \in \kappa_{0}(A)\right\} .
$$

Observe that $A$ has continuous-trace if and only if $\kappa(A) \subseteq m(A)$.

A $C^{*}$-algebra is called elementary if it is isomorphic to the compact operators on some Hilbert space. If $A$ is an elementary $C^{*}$-algebra and if $\pi: A \rightarrow \mathcal{K}\left(\mathcal{H}_{\pi}\right)$ and $\eta: A \rightarrow \mathcal{K}\left(\mathcal{H}_{\eta}\right)$ are two realizations of $A$ as the algebra of compact operators, then there is a unitary operator $U: \mathcal{H}_{\pi} \rightarrow \mathcal{H}_{\eta}$ such that $U \pi(a)=\eta(a) U$. It follows that the the usual traces on $B\left(\mathcal{H}_{\pi}\right)^{+}$and $B\left(\mathcal{H}_{\eta}\right)^{+}$satisfy $\operatorname{tr}(\pi(a))=\operatorname{tr}(\eta(a))$. Consequently, there is a canonical trace, tr, on an elementary $C^{*}$-algebra.

Lemma 6.3. Suppose that $A$ and $B$ are $C^{*}$-algebras and that $A \otimes_{\max } B$ is elementary. Then $A$ and $B$ are elementary.

We thank Siegfried Echterhoff for suggesting the following argument.

Proof. Since $A \otimes_{\max } B$ is simple, it coincides with the spatial tensor product $A \otimes_{\sigma} B$, and it will be more convenient to deal with the latter here. Suppose $A$ is not simple, and $I$ is a nonzero proper ideal in $A$. Then $I \otimes_{\sigma} B$ is a nonzero ideal in $A \otimes_{\sigma} B$. Let $\pi$ be a nondegenerate representation of $A$ with $\operatorname{ker}(\pi)=I$. Then if $\eta$ is any nonzero nondegenerate representation of $B, \pi \otimes \eta$ is a nonzero representation of $A \otimes_{\sigma} B$ such that $\pi \otimes \eta\left(I \otimes_{\sigma} B\right)=\{0\}$. This would imply that $A \otimes_{\sigma} B$ was not simple. Thus we can conclude that $A$, and by symmetry $B$, are simple.

Let $\pi$ and $\eta$ be irreducible representations of $A$ and $B$, respectively. Since $A$ and $B$ are simple, $\pi$ and $\eta$ are faithful, and $\pi \otimes \eta$ is a faithful irreducible representation 
of $A \otimes_{\sigma} B$ (cf., e.g., Corollary B.11 and Lemma B.36 of [25]). Thus $\pi \otimes \eta\left(A \otimes_{\sigma} B\right)$ is isomorphic to $\mathcal{K}\left(\mathcal{H}_{\pi} \otimes \mathcal{H}_{\eta}\right)$.

Now suppose there is an $a \in A$ such that $\pi(a)$ is not a compact operator on $\mathcal{H}_{\pi}$. There there is a sequence $\left\{h_{i}\right\}$ of unit vectors in $\mathcal{H}_{\pi}$ such that $\left\{\pi(a) h_{i}\right\}$ has no convergent subsequence. Choose $b \in B$ and $v \in \mathcal{H}_{\eta}$ such that $\eta(b) v \neq 0$. Then $\left\{\pi \otimes \eta(a \otimes b)\left(h_{i} \otimes v\right)\right\}$ has no convergent subsequence. This contradicts the compactness of $\pi \otimes \eta(a \otimes b)$. Therefore $\pi(A)$ is an irreducible subalgebra of $\mathcal{K}\left(\mathcal{H}_{\pi}\right)$, and hence $\pi(A) \cong \mathcal{K}\left(\mathcal{H}_{\pi}\right)$ by [1, Theorem 1.4.2].

Lemma 6.4. Suppose that $A$ and $B$ are $C^{*}$-algebras, that $a \in \kappa(A)^{+}$, and that $b \in \kappa(B)^{+}$. Then $a \otimes b \in \kappa\left(A \otimes_{\gamma} B\right)$ for any $C^{*}$-norm $\gamma$. Moreover, the image of $a \otimes b$ in $A \otimes_{X} B$ belongs to $\kappa\left(A \otimes_{X} B\right)$.

Proof. We can suppose that there are elements $a_{i} \in \kappa(A)_{0}$ and $b_{j} \in \kappa_{0}(B)$ such that

$$
a \leq \sum_{i=1}^{n} a_{i} \quad \text { and } \quad b \leq \sum_{j=1}^{m} b_{j} .
$$

Since it is not hard to see that $a \otimes b \leq \sum_{i, j} a_{i} \otimes b_{j}$, it suffices to prove the lemma in the case $a \in \kappa_{0}(A)$ and $b \in \kappa_{0}(B)$. Then there are $x \in A^{+}$and $y \in B^{+}$as well as $f, g \in C_{c}^{+}((0, \infty))$ such that $a=f(x)$ and $b=g(y)$. Let $h \in C_{c}^{+}((0, \infty))$ be such that $h(x)=1$ for all $x \in \operatorname{supp}(f) \cup \operatorname{supp}(g)$. Let $z=h(x) \otimes h(y)$. Note that $a \otimes b$ and $z$ are positive, and that $(a \otimes b) z=a \otimes b$. It follows that $a \otimes b$ belongs to $\kappa\left(A \otimes_{\gamma} B\right)$. But if $\pi: A \otimes_{\max } B \rightarrow A \otimes_{X} B$ is the quotient map, then $\pi(a \otimes b) \pi(z)=\pi(a \otimes b)$. The last assertion follows from this.

Lemma 6.5 (11, Lemma 2.4]). Let $A$ and $B$ be $C_{0}(X)$-algebras. Then the map $\left(a \otimes_{X} b\right)(x) \mapsto a(x) \otimes b(x)$ defines an isomorphism of the fibre $A \otimes_{X} B(x)$ onto $A(x) \otimes_{\max } B(x)$ for all $x \in X$.

Lemma 6.6. Suppose that $A$ and $B$ are in $\mathfrak{S}(X)$, and that $A \otimes_{X} B$ has continuous trace with spectrum $X$. Then $A$ and $B$ both have continuous trace with spectrum $X$.

Proof. We first show that $A$ is CCR and has Hausdorff spectrum $X$. By assumption $A \otimes_{X} B$ has continuous trace and is therefore CCR. Thus $\left(A \otimes_{X} B\right)(x)$ is elementary for all $x \in X$. Consequently Lemmas 6.5 and 6.3 imply that $A(x)$ and $B(x)$ are elementary for all $x \in X$. It follows that $\sigma_{A}: \operatorname{Prim}(A) \rightarrow X$ and $\sigma_{B}: \operatorname{Prim}(B) \rightarrow X$ are continuous bijections, and that $A$ and $B$ are CCR. We still need to see that $\sigma_{A}$ and $\sigma_{B}$ are homeomorphisms.

For each $a \in A$, let $f_{a}(x):=\|a(x)\|$. Note that $f_{a}$ is upper semicontinuous by Lemma 2.1(a). Now fix $a \in A$ and $y \in X$. Note that if $f_{a}(y)=0$, then $f_{a}$ is actually continuous at $y$. If $f_{a}(y) \neq 0$, then choose $b \in B$ such that $\|b(y)\| \neq$ 0 . Then since $A \otimes_{\sigma} B$ has Hausdorff spectrum, $f_{a \otimes b}=f_{a} f_{b}$ is continuous and strictly greater than zero near $y$. In particular, $f_{b}$ is strictly positive near $y$ and $f_{a}=f_{a} f_{b} / f_{b}$. Since the product of nonnegative lower semicontinuous functions is lower semicontinuous, the latter is lower semicontinuous (where it is defined). It follows that $f_{a}$ is continuous at $y$ in general. Thus $f_{a}$ is continuous for all $a \in A$, and it follows from Lemma 2.1(b) that $\sigma_{A}$ is open. Thus $A$ is CCR with spectrum homeomorphic to $X$ via $\sigma_{A}$. The same holds for $B$ by symmetry.

To show that $A$ has continuous trace, we will show that $\kappa(A)^{+} \subseteq m(A)$. Fix $a \in$ $\kappa(A)^{+}$and $y \in X$. Since $B$ is CCR and $\kappa(B)^{+}$is dense in $B^{+}$, there is a $b \in \kappa(B)^{+}$ 
such that $0<\operatorname{tr}(b(y))<\infty$. Since $x \mapsto \operatorname{tr}(b(x))$ is always lower semicontinuous [19, Proposition 4.4.9], it follows that $\operatorname{tr}(b(x)) \neq 0$ near $y$. Lemma 6.4 implies that $a \otimes b \in \kappa\left(A \otimes_{X} B\right)^{+}$. Since $m\left(A \otimes_{X} B\right)$ is dense by assumption, $a \otimes b$ is a continuous-trace element and

$$
g(x):=\operatorname{tr}(a \otimes b(x))
$$

defines a continuous function on $X$. Since the natural map from $\left(A \otimes_{X} B\right)(x)$ onto $A(x) \otimes_{\max } B(x)$ is an isomorphism (Lemma 6.5),

$$
\operatorname{tr}(a \otimes b(x))=\operatorname{tr}(a(x) \otimes b(x))=\operatorname{tr}(a(x)) \operatorname{tr}(b(x)) .
$$

Let $g_{a}(x):=\operatorname{tr}(a(x))$. Then $g_{a}=g / g_{b}$ near $y$. Since $g_{a}$ and $g_{b}$ are lower semicontinuous and since the product of nonnegative upper semicontinuous functions is always upper semicontinuous, it follows that $g_{a}$ is continuous at $y$. This proves that $\kappa(A)^{+} \subseteq m(A)$. Therefore $m(A)$ is dense and $A$ has continuous trace. The same holds for $B$ by symmetry.

Proof of Theorem [6.1. If $[A]$ has an inverse in $\mathrm{S}(X)$, then there is a $B \in \mathfrak{S}(X)$ such that $A \otimes_{X} B$ is Morita equivalent to $C_{0}(X)$ over $X$. In particular, $A \otimes_{X} B$ has continuous trace with spectrum $X$, and the theorem follows from Lemma 6.6

\section{REFERENCES}

1. William Arveson, An invitation to $C^{*}$-algebra, Graduate Texts in Mathematics, vol. 39, Springer-Verlag, New York, 1976. MR 58:23621

2. Étienne Blanchard, Tensor products of $C(X)$-algebras over $C(X)$, Astérisque 232 (1995), 81-92. MR 96m:46100

3. Déformations de $C^{*}$-algèbres de Hopf, Bull. Soc. Math. France 124 (1996), 141-215. MR 97f: 46092

4. Huu Hung Bui, Morita equivalence of crossed products, Ph.D. dissertation, University of New South Wales, August 1992.

5. François Combes, Crossed products and Morita equivalence, Proc. London Math. Soc. 49 (1984), 289-306. MR 86c:46081

6. David Crocker, Alexander Kumjian, Iain Raeburn, and Dana P. Williams, An equivariant Brauer group and actions of groups on $C^{*}$-algebras, J. Funct. Anal. 146 (1997), 151-184. MR 98j:46076

7. Raul E. Curto, Paul Muhly, and Dana P. Williams, Crossed products of strongly Morita equivalent $C^{*}$-algebras, Proc. Amer. Math. Soc. 90 (1984), 528-530. MR 85i:46083

8. Jacques Dixmier, $C^{*}$-algebras, North-Holland Mathematical Library, vol. 15, North-Holland, New York, 1977. MR 56:16388

9. Siegfried Echterhoff, Steven Kaliszewski, and Iain Raeburn, Crossed products by dual co-actions of groups and homogeneous spaces, J. Operator Theory 39 (1998), 151-176. MR 99h:46124

10. Siegfried Echterhoff and Dana P. Williams, Locally inner actions on $C_{0}(X)$-algebras, preprint, June 1997.

11. - Crossed products by $C_{0}(X)$-actions, J. Funct. Anal. 158 (1998), 113-151. CMP 98:17

12. Philip Green, The Brauer group of a commutative $C^{*}$-algebra, unpublished seminar notes, University of Pennsylvania, 1978.

13. - The local structure of twisted covariance algebras, Acta Math. 140 (1978), 191-250. MR 58:12376

14. Gennadi G. Kasparov, Equivariant KK-theory and the Novikov conjecture, Invent. Math. 91 (1988), 147-201. MR 88j:58123

15. Eberhard Kirchberg and Simon Wassermann, Operations on continuous bundles of $C^{*}$ algebras, Math. Ann. 303 (1995), 677-697. MR 96j:46057

16. Alexander Kumjian, Iain Raeburn, and Dana P. Williams, The equivariant Brauer groups of commuting free and proper actions are isomorphic, Proc. Amer. Math. Soc. 124 (1996), 809-817. MR 96f:46107 
17. May Nilsen, $C^{*}$-bundles and $C_{0}(X)$-algebras, Indiana Univ. Math. J. 45 (1996), $463-477$. MR 98e:46075

18. Judith A. Packer, Iain Raeburn, and Dana P. Williams, The equivariant Brauer groups of principal bundles, J. Operator Theory 36 (1996), 73-105. MR 98c:46123

19. Gert K. Pedersen, $C^{*}$-algebras and their automorphism groups, Academic Press, London, 1979. MR 81e:46037

20. John Quigg and Jack Spielberg, Regularity and hyporegularity in $C^{*}$-dynamical systems, Houston J. Math. 18 (1992), 139-152. MR 93c:46122

21. Iain Raeburn, Induced $C^{*}$-algebras and a symmetric imprimitivity theorem, Math. Ann. 280 (1988), 369-387. MR 90k:46144

22. Iain Raeburn and Jonathan Rosenberg, Crossed products of continuous-trace $C^{*}$-algebras by smooth actions, Trans. Amer. Math. Soc. 305 (1988), 1-45. MR 89e:46077

23. Iain Raeburn and Dana P. Williams, Pull-backs of $C^{*}$-algebras and crossed products by certain diagonal actions, Trans. Amer. Math. Soc. 287 (1985), 755-777. MR 86m:46054

24. - Dixmier-Douady classes of dynamical systems and crossed products, Canad. J. Math. 45 (1993), 1032-1066. MR 94k:46141

25. - Morita equivalence and continuous-trace $C^{*}$-algebras, Mathematical Surveys and Monographs, vol. 60, American Mathematical Society, Providence, RI, 1998. CMP 98:17

26. Marc A. Rieffel, Applications of strong Morita equivalence to transformation group $C^{*}$ algebras, Operator Algebras and Applications (Richard V. Kadison, ed.), Proc. Symp. Pure Math., vol. 38, Part I, Amer. Math. Soc., Providence, R.I., 1982, pp. 299-310. MR 84k:46046

27. and R. V. Kadison, eds.), Progr. Math., vol. 84, Birkhauser, Boston, 1988, Procceedings of the Japan-U.S. joint seminar, University of Pennsylvania, pp. 141-182. MR 92i:46079

28. _ Integrable and proper actions on $C^{*}$-algebras, and square integrable representations of groups, preprint, 1997.

29. Dana P. Williams, Transformation group $C^{*}$-algebras with continuous trace, J. Funct. Anal. 41 (1981), 40-76. MR 83c:46066

Department of Mathematics, Dartmouth College, Hanover, New Hampshire 037553551

Current address: Department of Mathematics, University of Denver, Denver, Colorado 80208

E-mail address: astrid@cs.du.edu

Department of Mathematics, University of Newcastle, Callaghan, New South Wales 2308, Australia

E-mail address: iain@math.newcastle.edu.au

Department of Mathematics, Dartmouth College, Hanover, New Hampshire 037553551

E-mail address: dana.williams@dartmouth.edu 